TRANSACTIONS OF THE

AMERICAN MATHEMATICAL SOCIETY

Volume 358, Number 1, Pages 183-214

S 0002-9947(04)03620-7

Article electronically published on December 28, 2004

\title{
A NEW APPROACH TO THE THEORY OF CLASSICAL HYPERGEOMETRIC POLYNOMIALS
}

\author{
JOSÉ MANUEL MARCO AND JAVIER PARCET
}

\begin{abstract}
In this paper we present a unified approach to the spectral analysis of a hypergeometric type operator whose eigenfunctions include the classical orthogonal polynomials. We write the eigenfunctions of this operator by means of a new Taylor formula for operators of Askey-Wilson type. This gives rise to some expressions for the eigenfunctions, which are unknown in such a general setting. Our methods also give a general Rodrigues formula from which several well-known formulas of Rodrigues-type can be obtained directly. Moreover, other new Rodrigues-type formulas come out when seeking for regular solutions of the associated functional equations. The main difference here is that, in contrast with the formulas appearing in the literature, we get non-ramified solutions which are useful for applications in combinatorics. Another fact, that becomes clear in this paper, is the role played by the theory of elliptic functions in the connection between ramified and non-ramified solutions.
\end{abstract}

\section{INTRODUCTION}

A large number of discrete problems in graph theory, group representation theory, discrete harmonic analysis or finite symmetric spaces involve the spectral analysis of certain difference operators. Namely, given a positive integer $d$ and denoting by $\mathrm{V}_{d}$ the space of functions $f:\{0,1, \ldots, d\} \rightarrow \mathbb{C}$, we are interested in the eigenfunctions of linear operators $\Lambda: \mathrm{V}_{d} \rightarrow \mathrm{V}_{d}$ of the following type:

$$
\Lambda f(t)=\sigma_{+}(t)(f(t+1)-f(t))-\sigma_{-}(t)(f(t)-f(t-1))
$$

where $\sigma_{ \pm} \in \mathrm{V}_{d}, \sigma_{-}(0)=\sigma_{+}(d)=0, \sigma_{-}(j) \neq 0$ if $1 \leq j \leq d$ and $\sigma_{+}(j) \neq 0$ if $0 \leq j \leq d-1$. For instance, the work [12] illustrates how these kinds of eigenvalue problems become useful in solving some a priori unrelated combinatorial problems. Let us write $\mathbb{P}_{k}[x]$ for the space of complex polynomials of degree $\leq k$ in one variable. Explicit solutions of this spectral analysis are available when there exists an injective mapping $x: t \in\{0,1, \ldots, d\} \mapsto x_{t} \in \mathbb{C}$ such that the following conditions hold:

(a) We have $x_{t+1}+x_{t-1}=f\left(x_{t}\right)$ for some $f \in \mathbb{P}_{1}[x]$ and $1 \leq t \leq d-1$.

(b) The subspaces $\mathrm{V}_{d, k}=\left\{f \circ x: f \in \mathbb{P}_{k}[x]\right\}$ are $\Lambda$-invariant for $0 \leq k \leq d-1$. By a well-known result of Leonard [9] see also the reference [5] — we have $(b \Rightarrow a)$ whenever $d>7$. When this is the case, the eigenfunctions of $\Lambda$ can be obtained by applying the theory of basic hypergeometric series. Alternatively, Nikiforov, Suslov and Uvarov study in 14 the spectral decomposition of $\Lambda$ using properties (a) and (b) and some specific methods. This gives rise to a whole new approach to the

Received by the editors July 17, 2003 and, in revised form, January 8, 2004.

2000 Mathematics Subject Classification. Primary 33D15, 33D45.

Key words and phrases. P-sequence, hypergeometric operator, Taylor and Rodrigues formula.

(C)2004 American Mathematical Society 
theory of classical orthogonal polynomials. Following any of these methods, two very relevant expressions for the eigenfunctions of $\Lambda$ can be obtained. First, as we have recalled above, we can write the eigenfunctions in terms of $q$-hypergeometric polynomials. Second, the eigenfunctions can be written in general by means of a Rodrigues-type formula. If we look at the approach which uses the theory of basic hypergeometric series, a long list of subcases must be checked. For instance, the reader is referred to the book of Bannai and Ito 4. Besides, in that reference the authors do not provide any Rodrigues-type formula for a problem which in essence is equivalent to the one considered here. On the other hand, in the approach of 14, the derivation of Rodrigues-type formulas is cumbersome. Moreover, at the very final stages, the authors need to invoke the Watson-Sears transformation and other formulas of the theory of $q$-series to get the hypergeometric expressions.

In this paper we propose an intermediate approach which, from our point of view, is quite simpler and provides a somehow natural classification of the whole problem which seems to be unknown. Throughout the paper, we shall also obtain some results and remarks which, as far as we know, are new in the theory. Following Nikiforov, Suslov and Uvarov, we shall study the eigenfunctions of $\Lambda$ with the aid of properties (a) and (b), so that the theory of basic hypergeometric series does not play a central role. However, in contrast with 14, we reformulate the operator $\Lambda$ in terms of operators of Askey-Wilson type, so that we work with a generalized hypergeometric operator L. By an Askey-Wilson operator D, we do not only mean the operators introduced by Richard Askey and James A. Wilson in 2]. Actually, we shall also be working with other kind of operators strongly related to those, such as the one introduced by Hahn. Besides, we shall use the companion operator S in the sense of Magnus [11. In particular, the hypergeometric operator we shall work with has the form

$$
\mathrm{L}=\sigma \mathrm{D}^{2}+\tau \mathrm{SD},
$$

where $\sigma \in \mathbb{P}_{2}[x]$ and $\tau \in \mathbb{P}_{1}[x]$. The operators $\mathrm{S}$ and $\mathrm{D}$ are defined by means of a two variable quadratic polynomial $\mathrm{P}$ which in our case will be symmetric. In our context, a translation of the difference operator $\Delta f(t)=f(t+1)-f(t)$ and the classical derivative will be particular cases. These Askey-Wilson-type operators act over polynomials - also over analytic functions - without any explicit reference to a particular sequence $x_{t}$ satisfying properties (a) and (b). Formulated in these terms, it turns out that the operator $\Lambda$ becomes more intrinsic and, as we shall see, this provides some relevant advantages.

In order to study the eigenvalue problem posed above, we previously state some results for the Askey-Wilson operators. We first give seven canonical forms under affine transformations which allow us to provide a very natural classification of the Askey-Wilson operators. We also develop the Leibniz and commutation formulas for the Askey-Wilson operator and its companion, the corresponding Taylor formula and Cauchy's integral formula. Results of this kind are interesting in their own right and can be used to obtain new proofs of well-known summation formulas for some basic hypergeometric series. We have not included here these applications since they will be the subject of a forthcoming publication [13. The use of these techniques, and mainly our Taylor formula, allow us to obtain simple expressions for the eigenfunctions of the hypergeometric operator L in a very general context; see Theorem 3.4. In particular, we do not need to invoke any Rodrigues-type formula or any result from the theory of basic hypergeometric polynomials, such 
as the Watson-Sears transformations used in [14. Moreover, the identities given in Theorem 3.4 seem to be unknown in such a general setting and, as we shall see, can be easily applied to particular cases. The expressions of the eigenfunctions in terms of $q$-hypergeometric functions arise from this result. On the other hand, Theorem 3.10 provides an algorithm to obtain Rodrigues-type formulas which depend on the resolution of certain functional equation. Our methods in Theorem 3.10 are closely related to those used in 14. However, the final result of Nikiforov, Suslov and Uvarov is not so clear, since they do not provide the relation with the AskeyWilson operators. Moreover, it is not easy to see the dependence of their Rodrigues formulas with respect to the function $x_{t}$ which appears in properties (a) and (b). Our Rodrigues formulas are also related to the ones obtained by Askey and Wilson in 2] for the Askey-Wilson polynomials.

The canonical Askey-Wilson operators mentioned above lead us to consider five canonical families of eigenvalue problems. For reasons that will become clear throughout the paper, we call these families as follows: continuous, arithmetic, quadratic, geometric and trigonometric. The corresponding eigenfunctions include as generic cases the Jacobi, Hahn, Wilson, $q$-Hahn and Askey-Wilson polynomials, respectively. We shall parameterize each family by means of one or two auxiliary polynomials. In the trigonometric case, we get a Laurent polynomial. Using this, we rewrite the expressions of Theorem 3.4 in terms of the basic hypergeometric functions. Besides, we will study the functional equation of Theorem 3.10 to obtain explicit Rodrigues formulas for each canonical case. Our approach looks mainly at the regularity of the solutions of the functional equation. This gives rise to new Rodrigues type formulas which do not appear in the literature. Quite surprisingly, apart from the continuous case, we can always obtain non-ramified solutions. More specifically, we get either meromorphic solutions or solutions with one essential singularity. In general, there is not uniqueness of solutions, so that we investigate a great variety of solutions. Although we shall only give explicit identities for some relevant generic cases, it will become clear that our methods can be applied in all cases. Moreover, in contrast with [14, our methods allow us to obtain such relations directly. That is, we do not need to take limits. We also point out that our Rodrigues formula for the Askey-Wilson polynomials is different from the one given by Askey and Wilson in 2. From our point of view, looking at the regularity of the functions appearing in Rodrigues formula, Askey and Wilson obtained ramified solutions while we get meromorphic ones. By using the non-uniqueness of the associated functional equation, we show how our Rodrigues formula can be used to get the orthogonality relations given in [2]. In fact, the techniques we develop here can be used to obtain discrete and absolutely continuous measures with respect to which the corresponding eigenfunctions become pairwise orthogonal. Besides, one of the most relevant features of our techniques is that the role of the theory of elliptic functions in the problem becomes quite clear. More concretely, our approach divides the analysis of Rodrigues-type formulas into two different parts. First, we seek for regular Rodrigues-type formulas avoiding ramified functions. Formulas of this kind are useful for applications in combinatorics. Second, with the aid of the theory of elliptic functions, we explore the non-uniqueness of solutions to obtain the original Rodrigues-type formulas given in [2].

Throughout this paper, we use the customary notation $[8$ for $q$-shifted factorials and basic hypergeometric series. We also use an auxiliary non-zero parameter $\lambda$ 
with $q=\lambda^{2}$. The restriction $|q|<1$ is only assumed in some explicit expressions. For instance, the main Theorems 3.4 and 3.10 hold without that restriction.

\section{P-Sequences}

In this section we introduce the notions of $\mathrm{P}$-sequence and $\mathrm{P}$-function associated to a given symmetric polynomial $\mathrm{P}$. As we shall see, these notions become very useful in order to give a somehow natural classification of the family of operators of Askey-Wilson type. Given $a, b, c$ complex numbers, let us consider the symmetric polynomial in two variables $\mathrm{P}(x, y)=x^{2}+y^{2}-2 a x y-2 b(x+y)+c$. For any such polynomial we have

$$
\mathrm{P}(x, y)=y^{2}-2 A(x) y+B(x),
$$

where $A(x)=a x+b$ and $B(x)=x^{2}-2 b x+c$. Let $\frac{1}{2} \mathbb{Z}$ stand for the set of integer and semi-integer numbers. A sequence of complex numbers $\left(x_{t}\right)$, with the index $t$ running over $\frac{1}{2} \mathbb{Z}$, will be called a $\mathrm{P}$-sequence if

$$
\mathrm{P}\left(x_{t}, y\right)=\left(y-x_{t+\frac{1}{2}}\right)\left(y-x_{t-\frac{1}{2}}\right) \quad \text { for all } t \in \frac{1}{2} \mathbb{Z} .
$$

If $\left(x_{t}\right)$ is a $\mathrm{P}$-sequence and $t_{0} \in \frac{1}{2} \mathbb{Z}$, the translated sequence $\left(y_{t}\right)$ with $y_{t}=x_{t-t_{0}}$ is also a $\mathrm{P}$-sequence. We shall say that $x_{0}$ is the base point of the $\mathrm{P}$-sequence $\left(x_{t}\right)$. Given a complex number $\xi$, a $\mathrm{P}$-sequence with base point $\xi$ can be constructed recursively. Moreover, this $\mathrm{P}$-sequence is unique once we have decided which root of $\mathrm{P}(\xi, y)$ is $x_{1 / 2}$ and which one is $x_{-1 / 2}$. In particular, we obtain two $\mathrm{P}$-sequences with base point $\xi$ which coincide when $\mathrm{P}(\xi, y)$ has a double root. If $\left(x_{t}\right)$ is such a $\mathrm{P}$-sequence, the other $\mathrm{P}$-sequence with base point $\xi$ is $\left(y_{t}\right)$, where $y_{t}=x_{-t}$.

Remark 1.1. Obviously, the symmetry of $\mathrm{P}$ plays an essential role in the iteration.

As we have seen, $\mathrm{P}$ can be regarded as a polynomial in the variable $y$ with coefficients depending on $x$. This allows us to consider the discriminant of $\mathrm{P}$ as a function of the variable $x$ which, up to a constant factor, has the form

$$
\delta(x)=A(x)^{2}-B(x)=\left(a^{2}-1\right) x^{2}+2 b(a+1) x+b^{2}-c .
$$

By the definition of P-sequence, the following identities hold for any $t \in \frac{1}{2} \mathbb{Z}$ :

$$
\begin{aligned}
A\left(x_{t}\right) & =\frac{1}{2}\left(x_{t+\frac{1}{2}}+x_{t-\frac{1}{2}}\right), \\
B\left(x_{t}\right) & =x_{t+\frac{1}{2}} x_{t-\frac{1}{2}}, \\
\delta\left(x_{t}\right) & =\frac{1}{4}\left(x_{t+\frac{1}{2}}-x_{t-\frac{1}{2}}\right)^{2} .
\end{aligned}
$$

Remark 1.2. (1) characterizes P-sequences with $\left(A\left(x_{t}\right), B\left(x_{t}\right)\right)$ or $\left(A\left(x_{t}\right), \delta\left(x_{t}\right)\right)$.

Each symmetric polynomial $\mathrm{P}(x, y)=x^{2}+y^{2}-2 a x y-2 b(x+y)+c$ is completely determined by its coefficients $a, b, c$. Given a parameterized curve

$$
\begin{aligned}
\gamma: \mathbb{R} & \longrightarrow \mathbb{C}^{3}, \\
s & \longmapsto(a(s), b(s), c(s)),
\end{aligned}
$$

we consider the polynomials $\mathrm{P}_{s}(x, y)=x^{2}+y^{2}-2 a(s) x y-2 b(s)(x+y)+c(s)$. Then, once we have fixed a complex number $x_{0}$, we can construct for any $s \in \mathbb{R}$ a $\mathrm{P}_{s}$-sequence $\left(x_{t}(s)\right)$ with base point $x_{0}$ such that the whole family of $\mathrm{P}_{s}$-sequences depend continuously on the parameter $s$. Namely, if $\delta_{s}$ stands for the discriminant 
of $\mathrm{P}_{s}$, we can choose the value of $\sqrt{\delta_{s}\left(x_{0}\right)}$ in such a way that we obtain a continuous function on $s$. This allows us to define

$$
x_{ \pm \frac{1}{2}}(s)=A_{s}\left(x_{0}\right) \pm \sqrt{\delta_{s}\left(x_{0}\right)}
$$

and then we construct the $\mathrm{P}_{s}$-sequence recursively. The continuous dependence along curves expressed above shows that any P-sequence with $a= \pm 1$ can be approximated by $\mathrm{P}$-sequences with $a \neq \pm 1$. This continuity argument will be very useful in the sequel and we shall use it in what follows with no further reference.

Let $\mathcal{P}$ be the set of polynomials $\mathrm{P}(x, y)=x^{2}+y^{2}-2 a x y-2 b(x+y)+c$. The set $\mathcal{P}$ is an affine subspace of the vector space $\mathbb{S P}_{2}[x, y]$ of symmetric polynomials in two complex variables having degree $\leq 2$. The affine group of the complex plane $\operatorname{Aff}(\mathbb{C})$ acts naturally on $\mathcal{P}$ by the action $\mathcal{A}_{1}: \operatorname{Aff}(\mathbb{C}) \times \mathcal{P} \rightarrow \mathcal{P}$ given by

$$
(g \cdot \mathrm{P})(x, y)=\zeta^{2} \mathrm{P}\left(g^{-1}(x), g^{-1}(y)\right),
$$

where $g(x)=\zeta x+\eta$. The parameter $a$, which will be called the main coefficient of $\mathrm{P}$, is invariant under the action of $\operatorname{Aff}(\mathbb{C})$ and the discriminant $\delta$ is transformed by the following rule:

$$
\delta^{g \cdot \mathrm{P}}(x)=\zeta^{2} \delta^{\mathrm{P}}\left(g^{-1}(x)\right) .
$$

The relation above defines another action $\mathcal{A}_{2}: \operatorname{Aff}(\mathbb{C}) \times \mathbb{P}_{2}[x] \rightarrow \mathbb{P}_{2}[x]$ on the vector space $\mathbb{P}_{2}[x]$ of complex polynomials in one variable with degree $\leq 2$. As we shall see throughout the paper, all the notions associated to the elements $\mathrm{P}$ of the set $\mathcal{P}$ are naturally preserved under the action of $\operatorname{Aff}(\mathbb{C})$. For instance, if we are given a P-sequence $\left(x_{t}\right)$, we have that $\left(g\left(x_{t}\right)\right)$ is a $(g \cdot \mathrm{P})$-sequence. A P-sequence $\left(x_{t}\right)$ is an even function of the variable $t$ if and only if its base point $x_{0}$ is a root of the discriminant $\delta$. For this reason we define the even points of $\mathrm{P}$ to be the roots of its discriminant $\delta$. The number of distinct even points of $\mathrm{P}$ will be denoted by $e v_{\mathrm{P}}$. Now we show that the main coefficient of $\mathrm{P}$ and the parameter $e v_{\mathrm{P}}$ are relevant invariants in the orbits of the action $\mathcal{A}_{1}$.

Proposition 1.3. Given a pair of symmetric polynomials $\mathrm{P}_{1}, \mathrm{P}_{2} \in \mathcal{P}$ with main coefficients $a_{j}$ and discriminants $\delta_{j}$ for $j=1,2$, the following are equivalent:

(a) The symmetric polynomials $\mathrm{P}_{1}$ and $\mathrm{P}_{2}$ belong to the same $\mathcal{A}_{1}$-orbit.

(b) The discriminants $\delta_{1}$ and $\delta_{2}$ have the same degree, $e v_{\mathrm{P}_{1}}=e v_{\mathrm{P}_{2}}$ and $a_{1}=$ $a_{2}$.

Proof. The implication ( $\mathrm{a} \Rightarrow \mathrm{b}$ ) follows from (2) and the recalled fact that the main coefficient remains invariant under the action of $\operatorname{Aff}(\mathbb{C})$. Let us see that $(b \Rightarrow a)$. If (b) holds, it is clear that $\delta_{1}$ and $\delta_{2}$ belong to the same $\mathcal{A}_{2}$-orbit. In particular, there exists $g_{12} \in \operatorname{Aff}(\mathbb{C})$ such that the polynomial $\mathrm{P}=g_{12} \cdot \mathrm{P}_{1}$ has discriminant $\delta_{2}$. If $a \neq-1$ this implies $\mathrm{P}=\mathrm{P}_{2}$ and we are done. Finally, for $a=-1$ we can always choose $g_{1}, g_{2} \in \operatorname{Aff}(\mathbb{C})$ such that

$$
\left(g_{j} \cdot \mathrm{P}_{j}\right)(x, y)=x^{2}+y^{2}+2 x y+c_{j} \quad \text { for } \quad j=1,2,
$$

where $c_{j}=1$ if $\delta_{j} \neq 0$ and $c_{j}=0$ otherwise. Since $\delta_{1}=0$ if and only if $\delta_{2}=0$ by hypothesis, $\mathrm{P}_{1}$ and $\mathrm{P}_{2}$ belong to the same $\mathcal{A}_{1}$-orbit and the proof is completed.

Using Proposition 1.3 we can write any polynomial $\mathrm{P}$ in $\mathcal{P}$, and its corresponding $\mathrm{P}$-sequences, in a canonical form. Namely, in the following table we write all the $\mathcal{A}_{1}$-orbits by considering all the possible combinations of $\mathcal{A}_{1}$-invariants: the main coefficient $a$, the degree $d g(\delta)$ of the discriminant and the number of distinct 
TABle I. Canonical P-sequences.

\begin{tabular}{|c|c|c|c|c|c|c|}
\multicolumn{9}{c}{$\boldsymbol{a}$} & $\boldsymbol{d}(\delta)$ & $\boldsymbol{e} \boldsymbol{v}_{\mathrm{P}}$ & Canonical form & P-sequence & $\mathbf{I}_{\mathrm{P}}$ \\
\hline $\mathbf{T}$ & $\neq \pm 1$ & 2 & 2 & $x^{2}+y^{2}-2 a x y+a^{2}-1$ & $\frac{1}{2}\left(\lambda^{2 t} u+\lambda^{-2 t} u^{-1}\right)$ & $\{ \pm 1\}$ \\
\hline $\mathbf{G}$ & $\neq \pm 1$ & 2 & 1 & $x^{2}+y^{2}-2 a x y$ & $\lambda^{ \pm 2 t} x_{0}$ & $G L(\mathbb{C})$ \\
\hline $\mathbf{Q}$ & 1 & 1 & 1 & $(x-y)^{2}-\frac{1}{2}(x+y)+\frac{1}{16}$ & $t^{2}+2 t u+u^{2}$ & 1 \\
\hline $\mathbf{A}$ & 1 & 0 & 0 & $(x-y)^{2}-\frac{1}{4}$ & $x_{0} \pm t$ & $\mathfrak{T}(\mathbb{C}) \rtimes\{ \pm 1\}$ \\
\hline $\mathbf{C}$ & 1 & $-\infty$ & $\infty$ & $(x-y)^{2}$ & $x_{0}$ & $\operatorname{Aff}(\mathbb{C})$ \\
\hline $\mathbf{O}$ & -1 & 0 & 0 & $(x+y)^{2}-\frac{1}{4}$ & $(-1)^{2 t}\left(x_{0} \pm t\right)$ & $\{ \pm 1\}$ \\
\hline $\mathbf{E}$ & -1 & $-\infty$ & $\infty$ & $(x+y)^{2}$ & $(-1)^{2 t} x_{0}$ & $G L(\mathbb{C})$ \\
\hline
\end{tabular}

even points $e v_{\mathrm{P}}$. We have chosen a canonical symmetric polynomial $\mathrm{P}$ in each $\mathcal{A}_{1}$-orbit. Then, we obtain the corresponding P-sequences. Our election for the canonical forms has given priority to the simplicity of the resulting $\mathrm{P}$-sequence and it is somehow arbitrary. Finally, recalling that $\operatorname{Aff}(\mathbb{C})$ can be regarded as the semidirect product $\mathfrak{T}(\mathbb{C}) \rtimes G L(\mathbb{C})$ where $\mathfrak{T}(\mathbb{C})$ is the group of translations in the complex plane, we include the isotropy subgroup $\mathbf{I}_{P}$ associated to each orbit. In the following table, we use a new parameter $\lambda \neq 0$ defined by the relation $\lambda=a \pm \sqrt{a^{2}-1}$. In other words,

$$
a=\frac{\lambda+\lambda^{-1}}{2}
$$

Obviously, the product of the two values assigned to $\lambda$ is 1 . Moreover, we have

$$
\begin{aligned}
\lambda=1 & \Leftrightarrow \quad a=1, \\
\lambda=-1 & \Leftrightarrow \quad a=-1 .
\end{aligned}
$$

As we have noticed above, these values of $\lambda$ can be regarded as limiting cases.

The first two canonical forms include infinitely many $\mathcal{A}_{1}$-orbits, one for each value of the main coefficient $a$. There are only seven inequivalent canonical forms. The capital letters in the left column are acronyms of the names we have adopted for the canonical forms. These names have been chosen attending at the shape of the canonical P-sequences: Trigonometric, Geometric, Quadratic, Arithmetic, Constant, Oscillating and Even. For some reason that will become clear later, we shall also refer to the Constant form as the Continuous canonical form.

Remark 1.4. It is clear that the polynomials belonging to the $\mathcal{A}_{1}$-orbits associated to the trigonometric canonical form are dense in $\mathcal{P}$. Therefore, the trigonometric canonical form is the most relevant one since any other canonical form can be regarded as a limit of families of polynomials in this particular orbit.

Remark 1.5. Taking affine transformations from the canonical P-sequences, it is not difficult to check that every P-sequence $\left(x_{t}\right)$ has one of the following forms:

- If $a \neq \pm 1$, then we have $x_{t}=k_{0}+k_{1} \lambda^{2 t}+k_{2} \lambda^{-2 t}$ where

$$
\begin{aligned}
x_{0} & =k_{0}+k_{1}+k_{2}, \\
b & =(1-a) k_{0}, \\
c & =2 b k_{0}+4 k_{1} k_{2}\left(a^{2}-1\right) .
\end{aligned}
$$


- If $a=1$, then we have $x_{t}=k_{0}+k_{1} t+k_{2} t^{2}$, where

$$
\begin{aligned}
x_{0} & =k_{0}, \\
b & =\frac{1}{4} k_{2}, \\
c & =\frac{1}{16} k_{2}^{2}-\frac{1}{4} k_{1}^{2}+k_{0} k_{2} .
\end{aligned}
$$

- If $a=-1$, then we have $x_{t}=k_{0}+(-1)^{2 t}\left(k_{1}+k_{2} t\right)$, where

$$
\begin{aligned}
x_{0} & =k_{0}+k_{1}, \\
b & =2 k_{0}, \\
c & =4 k_{0}^{2}-\frac{1}{4} k_{2}^{2} .
\end{aligned}
$$

These relations already appeared in the book 14] of Nikiforov, Suslov and Uvarov.

Proposition 1.6. Every $\mathrm{P}$-sequence $\left(x_{t}\right)$ satisfies the following relations, with the obvious limits for $\lambda= \pm 1$, for any $s \in \frac{1}{2} \mathbb{Z}$

$$
\begin{aligned}
& \frac{x_{t+s}+x_{t-s}}{2}=\frac{\lambda^{2 s}+\lambda^{-2 s}}{2} x_{t}+\frac{\lambda^{2 s}+\lambda^{-2 s}-2}{\lambda+\lambda^{-1}-2} b, \\
& x_{t+s}-x_{t-s}=\frac{\lambda^{2 s}-\lambda^{-2 s}}{\lambda-\lambda^{-1}}\left(x_{t+\frac{1}{2}}-x_{t-\frac{1}{2}}\right) .
\end{aligned}
$$

Proof. By continuity, it suffices to consider P-sequences with $a \neq \pm 1$. Moreover, by a translation in the variable $t$, we can assume that $t=0$. Finally, under these new hypothesis, the result follows easily from Remark 1.5 .

Corollary 1.7. Every $\mathrm{P}$-sequence $\left(x_{t}\right)$ satisfies the following relations:

(a) $x_{t+1}-x_{t-1}=2 a\left(x_{t+\frac{1}{2}}-x_{t-\frac{1}{2}}\right)$.

(b) $8 a \delta\left(x_{t}\right)=\left(x_{t+1}-x_{t-1}\right)\left(x_{t+\frac{1}{2}}-x_{t-\frac{1}{2}}\right)$.

(c) $x_{t+1}-2 x_{t}+x_{t-1}=4(a+1)\left(A\left(x_{t}\right)-x_{t}\right)$.

Given a symmetric polynomial $\mathrm{P} \in \mathcal{P}$, an entire function $\theta: \mathbb{C} \rightarrow \mathbb{C}$ will be called a P-function if the sequence

$$
\ldots \theta(z-1), \theta\left(z-\frac{1}{2}\right), \theta(z), \theta\left(z+\frac{1}{2}\right), \theta(z+1), \ldots
$$

is a $\mathrm{P}$-sequence for any $z \in \mathbb{C}$. To be consistent with our notation, we shall write $\theta_{t}$ for $\theta(t)$. P-functions and P-sequences can be treated in the same fashion. For instance, an entire function $\theta: \mathbb{C} \rightarrow \mathbb{C}$ is a P-function if and only if the following relations hold for any $t \in \mathbb{C}$ :

$$
A\left(\theta_{t}\right)=\frac{1}{2}\left(\theta_{t+\frac{1}{2}}+\theta_{t-\frac{1}{2}}\right) \quad \text { and } \quad B\left(\theta_{t}\right)=\theta_{t+\frac{1}{2}} \theta_{t-\frac{1}{2}} .
$$

Remark 1.8. In what follows, when dealing with P-functions, we shall write $\lambda^{t}$ as an abbreviation of $e^{t \omega}$ for some election $\omega$ of the $\operatorname{logarithm} \log \lambda$ and any complex number $t$. We shall also make this slight abuse of notation for $q^{t}$, where $q=\lambda^{2}$.

\section{TAYLOR FORMUla FOR THE ASKey-Wilson OPERATOR}

In this section we develop a Taylor formula for operators of Askey-Wilson type, which will we shall apply in Sections 3 and 4 to give explicit expressions for the polynomic eigenfunctions of the associated hypergeometric operator. 
2.1. Operators $\mathrm{S}$ and $\mathrm{D}$. If $\mathbb{P}[x]$ stands for the space of complex polynomials in one variable, let us denote by $\mathbb{P}_{k}[x]$ the subspace of polynomials of degree $\leq k$. Given $f \in \mathbb{P}[x]$, we define $f_{d}(x, y)$ as the polynomial which coincides with

$$
\frac{f(x)-f(y)}{x-y}
$$

whenever $x \neq y$. We also introduce the polynomial $f_{s}(x, y)=\frac{1}{2}(f(x)+f(y))$. Both $f_{s}(x, y)$ and $f_{d}(x, y)$ are symmetric polynomials in two variables. On the other hand, given a symmetric polynomial $\mathrm{P}(x, y)=x^{2}+y^{2}-2 a x y-2 b(x+y)+c$, we shall denote by $u(x)$ and $v(x)$ the roots of $\mathrm{P}(x, y)$ in the variable $y$. That is, we have $\mathrm{P}(x, y)=(y-u(x))(y-v(x))$ for all $x \in \mathbb{C}$. Then we define the operators $\mathrm{S}$ and $\mathrm{D}$ as follows:

$$
\mathrm{S} f(x)=f_{s}(u(x), v(x)) \quad \text { and } \quad \mathrm{D} f(x)=f_{d}(u(x), v(x)) .
$$

As recalled by Magnus [1], the divided difference operator D is essentially the Askey-Wilson operator while $\mathrm{S}$ will be called the companion operator to D. Any symmetric polynomial in two variables provides a polynomial in the variable $x$ when it is evaluated at $(u(x), v(x))$. In particular, we have that $\mathrm{S} f \in \mathbb{P}[x]$ and $\mathrm{D} f \in \mathbb{P}[x]$ for any $f \in \mathbb{P}[x]$. That is, $\mathrm{S}, \mathrm{D} \in \operatorname{End}(\mathbb{P}[x])$ are linear mappings in $\mathbb{P}[x]$.

Remark 2.1. S and D are naturally transformed under the action of Aff( $\mathbb{C})$. That is, if $\mathrm{S}^{\mathrm{P}}$ and $\mathrm{D}^{\mathrm{P}}$ stand for the operators associated to the symmetric polynomial $\mathrm{P}$ and we take some $g=(\eta, \zeta) \in \mathfrak{T}(\mathbb{C}) \rtimes G L(\mathbb{C})=\operatorname{Aff}(\mathbb{C})$, then we have

$$
g \mathrm{~S}^{\mathrm{P}} g^{-1}=\mathrm{S}^{g \cdot \mathrm{P}} \quad \text { and } \quad g \mathrm{D}^{\mathrm{P}} g^{-1}=\zeta \mathrm{D}^{g \cdot \mathrm{P}},
$$

where we identify $g \in \operatorname{Aff}(\mathbb{C})$ with the induced mapping $(g \cdot f)(x)=f\left(g^{-1}(x)\right)$.

Proposition 2.2. We have $\mathrm{S}\left(\mathbb{P}_{k}[x]\right) \subset \mathbb{P}_{k}[x]$ and $\mathrm{D}\left(\mathbb{P}_{k}[x]\right) \subset \mathbb{P}_{k-1}[x]$ for $k \geq 1$. Moreover, if $f(x)=x^{k}$ we obtain the following expressions:

$$
\begin{aligned}
\mathrm{S} f(x) & =\frac{\lambda^{k}+\lambda^{-k}}{2} x^{k}+\ldots, \\
\operatorname{D} f(x) & =\frac{\lambda^{k}-\lambda^{-k}}{\lambda-\lambda^{-1}} x^{k-1}+\ldots,
\end{aligned}
$$

where the dots stand for terms of lower degree. If $f \in \mathbb{P}_{0}[x], \mathrm{S} f=f$ and $\mathrm{D} f=0$.

Proof. The action of $\mathrm{S}$ and $\mathrm{D}$ on $\mathbb{P}_{0}[x]$ is trivial. Now, let $f(x)=x^{k}$ for some $k \geq 1$. Then, since the roots of $\mathrm{P}(x, y)$ are given by $A(x) \pm \sqrt{\delta(x)}$, we have

$$
\mathrm{D} f(x)=\frac{(A(x)+\sqrt{\delta(x)})^{k}-(A(x)-\sqrt{\delta(x)})^{k}}{2 \sqrt{\delta(x)}}
$$

whenever $\delta(x) \neq 0$. The binomial theorem gives

$$
\begin{aligned}
\mathrm{D} f(x) & =\frac{1}{2} \sum_{j=0}^{k}\left(\begin{array}{l}
k \\
j
\end{array}\right) A(x)^{k-j}(\sqrt{\delta(x)})^{j-1}\left(1-(-1)^{j}\right) \\
& =\frac{x^{k-1}}{2 \sqrt{a^{2}-1}} \sum_{j=0}^{k}\left(\begin{array}{l}
k \\
j
\end{array}\right) a^{k-j}\left(\sqrt{a^{2}-1}\right)^{j}\left(1-(-1)^{j}\right)+\ldots \\
& =\frac{x^{k-1}}{2 \sqrt{a^{2}-1}}\left[\left(a+\sqrt{a^{2}-1}\right)^{k}-\left(a-\sqrt{a^{2}-1}\right)^{k}\right]+\ldots
\end{aligned}
$$


where the dots stand for terms of lower degree. This new expression is now valid for any $x \in \mathbb{C}$. In particular, we have obtained

$$
\mathrm{D} f(x)=\frac{\lambda^{k}-\lambda^{-k}}{\lambda-\lambda^{-1}} x^{k-1}+\ldots
$$

Similar arguments provide the expression given above for $\mathrm{S} f(x)$.

Remark 2.3. Both operators S and D depend polynomially on P. In particular, Proposition 2.2 and the forthcoming expressions hold for $\lambda= \pm 1$ by taking the obvious limits. This follows from a simple continuity argument.

Proposition 2.4. S and D satisfy the following Leibniz rules:

$$
\begin{aligned}
& \mathrm{S}(f g)(x)=\mathrm{S} f(x) \mathrm{S} g(x)+\delta(x) \mathrm{D} f(x) \mathrm{D} g(x), \\
& \mathrm{D}(f g)(x)=\mathrm{D} f(x) \mathrm{S} g(x)+\mathrm{S} f(x) \mathrm{D} g(x) .
\end{aligned}
$$

Proof. Both expressions are straightforward and we leave them to the reader.

If $\left(x_{t}\right)$ is a $\mathrm{P}$-sequence, then we can use our definitions of the operators $\mathrm{S}$ and $\mathrm{D}$ to obtain the relations

$$
\begin{aligned}
& \mathrm{S} f\left(x_{t}\right)=f_{s}\left(x_{t+\frac{1}{2}}, x_{t-\frac{1}{2}}\right)=\frac{f\left(x_{t+\frac{1}{2}}\right)+f\left(x_{t-\frac{1}{2}}\right)}{f\left(x_{t+\frac{1}{2}}\right)-f\left(x_{t-\frac{1}{2}}\right)}, \\
& \operatorname{Df}\left(x_{t}\right)=f_{d}\left(x_{t+\frac{1}{2}}, x_{t-\frac{1}{2}}\right)=\frac{x_{t-\frac{1}{2}}}{x_{t+\frac{1}{2}}} .
\end{aligned}
$$

Also, it is not difficult to check the validity of the identities

$$
\begin{aligned}
& \mathrm{S} f\left(x_{t}\right)=f\left(x_{t+\frac{1}{2}}\right)-\frac{x_{t+\frac{1}{2}}-x_{t-\frac{1}{2}}}{2} \mathrm{D} f\left(x_{t}\right), \\
& \mathrm{S} f\left(x_{t}\right)=f\left(x_{t-\frac{1}{2}}\right)+\frac{x_{t+\frac{1}{2}}-x_{t-\frac{1}{2}}}{2} \mathrm{D} f\left(x_{t}\right) .
\end{aligned}
$$

Proposition 2.5. S and $\mathrm{D}$ satisfy the relations

$$
\begin{aligned}
\operatorname{DS} f(x) & =(a+1)(A(x)-x) \mathrm{D}^{2} f(x)+a \operatorname{SD} f(x), \\
\mathrm{S}^{2} f(x) & =a \delta(x) \mathrm{D}^{2} f(x)+(a+1)(A(x)-x) \operatorname{SD} f(x)+f(x) .
\end{aligned}
$$

Proof. Given a complex number $x$, let $\left(x_{t}\right)$ be a P-sequence with base point $x$. By continuity, we can assume that

$$
x_{t+\frac{1}{2}} \neq x_{t-\frac{1}{2}} \quad \text { for } \quad t=0, \pm \frac{1}{2} .
$$


Under these assumptions, we can use Corollary 1.7 and (3) to obtain

$$
\begin{aligned}
\operatorname{DS} f(x) & =\frac{1}{x_{\frac{1}{2}}-x_{-\frac{1}{2}}}\left(\frac{f\left(x_{1}\right)+f(x)}{2}-\frac{f(x)+f\left(x_{-1}\right)}{2}\right), \\
4 a \operatorname{SD} f(x) & =\frac{x_{1}-x_{-1}}{x_{\frac{1}{2}}-x_{-\frac{1}{2}}}\left(\frac{f\left(x_{1}\right)-f(x)}{x_{1}-x}+\frac{f(x)-f\left(x_{-1}\right)}{x-x_{-1}}\right), \\
(a+1)(A(x)-x) \mathrm{D}^{2} f(x) & =\frac{x_{1}-2 x+x_{-1}}{4\left(x_{\frac{1}{2}}-x_{-\frac{1}{2}}\right)}\left(\frac{f\left(x_{1}\right)-f(x)}{x_{1}-x}-\frac{f(x)-f\left(x_{-1}\right)}{x-x_{-1}}\right), \\
(a+1)(A(x)-x) \operatorname{SD} f(x) & =\frac{x_{1}-2 x+x_{-1}}{8}\left(\frac{f\left(x_{1}\right)-f(x)}{x_{1}-x}+\frac{f(x)-f\left(x_{-1}\right)}{x-x_{-1}}\right), \\
a \delta(x) \mathrm{D}^{2} f(x) & =\frac{x_{1}-x_{-1}}{8}\left(\frac{f\left(x_{1}\right)-f(x)}{x_{1}-x}-\frac{f(x)-f\left(x_{-1}\right)}{x-x_{-1}}\right), \\
\mathrm{S}^{2} f(x) & =\frac{f\left(x_{1}\right)+f(x)}{4}+\frac{f(x)+f\left(x_{-1}\right)}{4} .
\end{aligned}
$$

At this point it is easy to check the stated relations. This completes the proof.

Remark 2.6. Once we have introduced the operators $\mathrm{S}$ and $\mathrm{D}$, we are in a position to justify some terminology introduced above. It follows from Table I that the $\mathrm{P}$-sequences of the canonical form $\mathbf{C}$ are constant. In particular, $\mathrm{S}$ is the identity operator while $\mathrm{D}$ is given by the classical derivative

$$
\mathrm{D} f=\frac{d f}{d x} \text {. }
$$

This is why we have decided to call this canonical form the continuous form. On the other hand, the P-sequences of the arithmetic canonical form are given by $x_{t}=x_{0} \pm t$. In particular, $\mathrm{S}$ can be regarded as an arithmetic mean while $\mathrm{D}$ can be rewritten in terms of the classical operator $\Delta f(x)=f(x+1)-f(x)$ as follows:

$$
\mathrm{D} f(x)=\Delta f\left(x-\frac{1}{2}\right) .
$$

In summary, these canonical forms can be regarded as the 'classical' forms.

Remark 2.7. By Proposition 2.5 we can characterize those polynomials $\mathrm{P}$ for which we have $\mathrm{SD}=\mathrm{DS}$. Namely, the operators $\mathrm{S}$ and D commute if and only if the coefficients of $\mathrm{P}$ satisfy $a=1$ and $b=0$. In other words, this happens only for the classical forms described in Remark 2.6.

2.2. Taylor formula. In order to construct the polynomials which will appear in the Taylor formula for the Askey-Wilson operator, we shall need the following lemma, which is a simple consequence of Proposition 1.6

Lemma 2.8. Given a $\mathrm{P}$-sequence $\left(x_{t}\right)$, let us consider the sequence $y_{t}=x_{j t}$ for some integer number $j \in \mathbb{Z}$. Then we have

$$
\frac{y_{t+\frac{1}{2}}+y_{t-\frac{1}{2}}}{2}=a_{j} y_{t}+b_{j} \quad \text { and } \quad\left(y_{t+\frac{1}{2}}-y_{t-\frac{1}{2}}\right)^{2}=4\left(\frac{\lambda^{j}-\lambda^{-j}}{\lambda-\lambda^{-1}}\right)^{2} \delta\left(y_{t}\right),
$$

where the coefficients $a_{j}$ and $b_{j}$ are given by

$$
a_{j}=\frac{\lambda^{j}+\lambda^{-j}}{2} \quad \text { and } \quad b_{j}=\frac{\lambda^{j}+\lambda^{-j}-2}{\lambda+\lambda^{-1}-2} b .
$$


It turns out by Lemma 2.8 that $\left(y_{t}\right)$ is a $\mathrm{P}_{j}$-sequence, where $\mathrm{P}_{j}$ is the following symmetric polynomial:

$$
\mathrm{P}_{j}(x, y)=\left(y-a_{j} x-b_{j}\right)^{2}-\left(\frac{\lambda^{j}-\lambda^{-j}}{\lambda-\lambda^{-1}}\right)^{2} \delta(x) \quad \text { with } \quad \delta_{j}(x)=\left(\frac{\lambda^{j}-\lambda^{-j}}{\lambda-\lambda^{-1}}\right)^{2} \delta(x) .
$$

The symmetric polynomials $\mathrm{P}_{j}$ are naturally transformed under the action of $\mathrm{Aff}(\mathbb{C})$. Namely, we have

$$
(g \cdot \mathrm{P})_{j}(x, y)=\left(g \cdot \mathrm{P}_{j}\right)(x, y) .
$$

Given a positive integer $k \geq 1$, we define the polynomials $\Phi_{k}(x, y)$ as follows:

$$
\Phi_{2 k}(x, y)=\prod_{j=1}^{k} \mathrm{P}_{2 j-1}(x, y) \quad \text { and } \quad \Phi_{2 k+1}(x, y)=(y-x) \prod_{j=1}^{k} \mathrm{P}_{2 j}(x, y) .
$$

We set $\Phi_{0}(x, y)=1$ and $\Phi_{1}(x, y)=y-x$. In particular, $\Phi_{k}(x, y)=(-1)^{k} \Phi_{k}(y, x)$ for all $k \geq 0$. Moreover, given two P-sequences $\left(x_{t}\right)$ and $\left(y_{t}\right)$, it follows by Lemma 2.8 that

$$
\Phi_{k}\left(x_{0}, y_{0}\right)=\prod_{j=0}^{k-1}\left(y_{0}-x_{j-\frac{k-1}{2}}\right)=\prod_{j=0}^{k-1}\left(y_{j-\frac{k-1}{2}}-x_{0}\right) \quad \text { for } \quad k \geq 1 .
$$

Remark 2.9. The polynomials $\Phi_{k}$ depend continuously on the coefficients $a, b, c$. In particular, once again we can take limits $\lambda \rightarrow \pm 1$ in the forthcoming expressions.

Lemma 2.10. The following relations hold for any $k \geq 1$ :

$$
\begin{aligned}
& \mathrm{D} \Phi_{k}(\cdot, y)=\frac{\lambda^{-k}-\lambda^{k}}{\lambda-\lambda^{-1}} \Phi_{k-1}(\cdot, y), \\
& \mathrm{D} \Phi_{k}(x, \cdot)=\frac{\lambda^{k}-\lambda^{-k}}{\lambda-\lambda^{-1}} \Phi_{k-1}(x, \cdot) .
\end{aligned}
$$

Moreover, for $k=0$ we have the relations $\mathrm{D}_{0}(\cdot, y)=\mathrm{D} \Phi_{0}(x, \cdot)=0$.

Proof. Given a complex number $x$, we consider a P-sequence with base point $x$. Moreover, by continuity we can assume that $x_{1 / 2} \neq x_{-1 / 2}$. In that case, it is not difficult to check that

$$
\frac{\Phi_{k}\left(x_{1 / 2}, y\right)-\Phi_{k}\left(x_{-1 / 2}, y\right)}{x_{1 / 2}-x_{-1 / 2}}=\frac{x_{-k / 2}-x_{k / 2}}{x_{1 / 2}-x_{-1 / 2}} \Phi_{k-1}\left(x_{0}, y\right)
$$

The first relation is then obtained by Proposition 1.6. The second relation follows by the first relation and the identity $\Phi_{k}(x, y)=(-1)^{k} \Phi_{k}(y, x)$. Finally, when $k=0$ the stated relations are obvious. This completes the proof.

Lemma 2.11. The linear operator $\partial_{k}: \mathbb{P}_{d}[x] \rightarrow \mathbb{P}_{d-k}[x]$ defined by

$$
\partial_{k} f(x)=\left(\prod_{j=0}^{k-1} \frac{\lambda-\lambda^{-1}}{\lambda^{k-j}-\lambda^{j-k}}\right) \mathrm{D}^{k} f(x) \quad \text { for } \quad|\lambda| \neq 1
$$

has a continuous extension for any $\lambda$ which depends polynomially on $\mathrm{P}$.

Proof. From Lemma 2.10, the following relation holds whenever $r \geq k$ and the parameter $\lambda$ satisfies $|\lambda| \neq 1$ :

$$
\partial_{k} \Phi_{r}(x, \cdot)=\left(\prod_{j=0}^{k-1} \frac{\lambda^{r-j}-\lambda^{j-r}}{\lambda^{k-j}-\lambda^{j-k}}\right) \Phi_{r-k}(x, \cdot) .
$$


Moreover, $\partial_{k} \Phi_{r}(x, \cdot)$ vanishes for $r<k$. By a well-known result of $q$-combinatorics (see for instance [10]) the rational function which appears in (6) is a symmetric polynomial in $\lambda$ and $\lambda^{-1}$. In particular, the function $\partial_{k} \Phi_{r}(x, \cdot)$ can be written as a polynomial in $a=\frac{1}{2}\left(\lambda+\lambda^{-1}\right), b$ and $c$. Since for any fixed $x$, the family $\Phi_{0}(x, \cdot), \Phi_{1}(x, \cdot), \ldots, \Phi_{d}(x, \cdot)$ is a basis of $\mathbb{P}_{d}[x]$, the proof is completed.

Remark 2.12. The operator $\partial_{k}$ defined in Lemma 2.11 can be regarded as the analog of the divided power of the $k$-th derivative $\partial^{[k]}$, which appears in finite field theory.

Remark 2.13. Taking $q=\lambda^{2}$, the rational function appearing in the proof of Lemma 2.11 can be rewritten in terms of a $q$-binomial coefficient

$$
\prod_{j=0}^{k-1} \frac{\lambda^{r-j}-\lambda^{j-r}}{\lambda^{k-j}-\lambda^{j-k}}=\lambda^{-k(r-k)}\left[\begin{array}{l}
r \\
k
\end{array}\right]_{q}, \quad \text { where } \quad\left[\begin{array}{l}
r \\
k
\end{array}\right]_{q}=\frac{(q ; q)_{r}}{(q ; q)_{k}(q ; q)_{r-k}} .
$$

The following result constitutes a Taylor formula for the Askey-Wilson operator. As we shall immediately see, it generalizes the continuous and discrete classical expressions of this formula.

Theorem 2.14. If $\left(x_{t}\right)$ is a $\mathrm{P}$-sequence and $f \in \mathbb{P}_{r}[x]$, then we have

$$
f(y)=\sum_{k=0}^{r} \partial_{k} f\left(x_{k / 2}\right) \prod_{j=0}^{k-1}\left(y-x_{j}\right) .
$$

Proof. There exists a family of complex coefficients $\lambda_{0}, \lambda_{1}, \ldots, \lambda_{r}$ such that

$$
f(y)=\sum_{k=0}^{r} \lambda_{k} \prod_{j=0}^{k-1}\left(y-x_{j}\right)
$$

On the other hand, since $\prod_{j=0}^{k-1}\left(y-x_{j}\right)=\Phi_{k}\left(x_{(k-1) / 2}, y\right)$, formula (마) gives

$$
\partial_{j} f(y)=\sum_{k=j}^{r} \lambda_{k}\left(\prod_{i=0}^{j-1} \frac{\lambda^{k-i}-\lambda^{i-k}}{\lambda^{j-i}-\lambda^{i-j}}\right) \Phi_{k-j}\left(x_{(k-1) / 2}, y\right) .
$$

By identity (5), if $k>j$, then $\Phi_{k-j}\left(x_{(k-1) / 2}, y\right)$ vanishes at

$$
y=x_{i+\frac{j}{2}} \quad \text { for } \quad i=0,1, \ldots, k-j-1 .
$$

In particular, evaluating our expression for $\partial_{j} f(y)$ at the point $y=x_{j / 2}$, we obtain the identity $\lambda_{k}=\partial_{k} f\left(x_{k / 2}\right)$ as we wanted. This completes the proof.

Remark 2.15. The classical continuous and discrete Taylor formulas are particular cases of Theorem 2.14. Namely, if $\mathrm{P}(x, y)=x^{2}+y^{2}-2 x y$ the P-sequences are constant and the operator $\mathrm{D}$ coincides with the classical derivative, as it was recalled in Remark 2.6. Therefore, Theorem 2.14 takes the classical form

$$
f(y)=\sum_{k=0}^{r} \frac{f^{(k)}(x)}{k !}(y-x)^{k} .
$$

On the other hand, if $\mathrm{P}(x, y)=(x-y)^{2}-\frac{1}{4}$, we know that the $\mathrm{P}$-sequences have the form $x_{0} \pm t$. Thus, using the classical notation $[t]_{k}=t(t-1) \cdots(t-k+1)$ and 
$\Delta f(x)=f(x+1)-f(x)$, we obviously have

$$
\Phi_{k}(x, y)=\left[y-x+\frac{k-1}{2}\right]_{k} \quad \text { and } \quad \partial_{k} f(x)=\frac{1}{k !} \Delta^{k} f\left(x-\frac{k}{2}\right) .
$$

In particular, Theorem 2.14 provides the discrete Taylor formula

$$
f(y)=\sum_{k=0}^{r} \Delta^{k} f(x)\left(\begin{array}{c}
y-x \\
k
\end{array}\right) .
$$

2.3. Some remarks for analytic functions. Given an open subset $\Omega$ of the complex plane, we denote the space of analytic functions in $\Omega$ by $\mathcal{H}(\Omega)$. Also, $\gamma \simeq 0(\bmod \Omega)$ means that $\gamma$ is a cycle in $\Omega$ homologous to zero with respect to $\Omega$. Finally, given $z \in \Omega, \operatorname{Ind}(\gamma, z)$ denotes the index of $z$ with respect to $\gamma$.

Lemma 2.16. Given an open subset $\Omega$ of the complex plane, let us denote by $\Delta_{\Omega}$ the diagonal of $\Omega \times \Omega$. Then, for any $f \in \mathcal{H}(\Omega)$, the function

$$
f_{d}(u, v)=\frac{f(u)-f(v)}{u-v} \quad \text { for } \quad(u, v) \in(\Omega \times \Omega) \backslash \Delta_{\Omega}
$$

can be continuously extended to an analytic function $f_{d}: \Omega \times \Omega \rightarrow \mathbb{C}$. Moreover, if $\gamma \simeq 0(\bmod \Omega)$ and $\operatorname{Ind}(\gamma, u)=\operatorname{Ind}(\gamma, v)=1$, then we have

$$
f_{d}(u, v)=\frac{1}{2 \pi i} \int_{\gamma} \frac{f(y)}{(y-u)(y-v)} d y .
$$

Proof. It is a simple consequence of Cauchy's integral formula.

Let us consider the set $\Omega^{(1)}=\{x \in \mathbb{C}: A(x) \pm \sqrt{\delta(x)} \in \Omega\}$. By Lemma 2.16, we can define the operator $\mathrm{D}: \mathcal{H}(\Omega) \rightarrow \mathcal{H}\left(\Omega^{(1)}\right)$ as follows:

$$
\mathrm{D} f(x)=f_{d}(A(x)+\sqrt{\delta(x)}, A(x)-\sqrt{\delta(x)})=\frac{1}{2 \pi i} \int_{\gamma} \frac{f(y)}{\mathrm{P}(x, y)} d y,
$$

with $\gamma \simeq 0(\bmod \Omega)$ and $\operatorname{Ind}(\gamma, A(x) \pm \sqrt{\delta(x)})=1$. It is clear that (7) extends the original definition of the Askey-Wilson operator D. Moreover, we can also extend the definition of the companion operator S. Namely, if $f \in \mathcal{H}(\Omega), x_{0} \in \Omega^{(1)}$ and $\delta\left(x_{0}\right) \neq 0$, the function

$\mathrm{S} f(x)=f_{s}(A(x)+\sqrt{\delta(x)}, A(x)-\sqrt{\delta(x)})=\frac{f(A(x)+\sqrt{\delta(x)})+f(A(x)-\sqrt{\delta(x)})}{2}$ is obviously analytic for $x$ in a neighborhood of $x_{0}$. Besides, $\mathrm{S} f$ is continuous at $x_{0}$ when $\delta\left(x_{0}\right)=0$. Thus, the roots of $\delta$ are removable singularities and $\mathrm{S} f$ becomes analytic in $\Omega^{(1)}$. If we define recursively the sets

$$
\Omega^{(k+1)}=\left\{x \in \mathbb{C}: A(x) \pm \sqrt{\delta(x)} \in \Omega^{(k)}\right\} \quad \text { with } \quad \Omega^{(0)}=\Omega,
$$

we can consider the iterated operators $\mathrm{S}^{k}, \mathrm{D}^{k}: \mathcal{H}(\Omega) \rightarrow \mathcal{H}\left(\Omega^{(k)}\right)$. An open subset $\Omega$ of the complex plane will be called P-invariant if, for any P-sequence with base point $x_{0} \in \Omega$, we have $x_{ \pm 1 / 2} \in \Omega$. In other words, if $\Omega \subset \Omega^{(k)}$ for any positive integer $k \geq 1$. If $\Omega$ is P-invariant, we deduce that $\mathrm{S}^{k} f \in \mathcal{H}(\Omega)$ and $\mathrm{D}^{k} f \in \mathcal{H}(\Omega)$ for any function $f$ analytic in $\Omega$ and any positive integer $k$.

Remark 2.17. Similarly, if $\mathcal{M}(\Omega)$ stands for the space of meromorphic functions in $\Omega$, we can extend the previous operators so that

$$
\mathrm{S}^{k}, \mathrm{D}^{k}: \mathcal{M}(\Omega) \rightarrow \mathcal{M}\left(\Omega^{(k)}\right) .
$$


Furthermore, if $\Omega$ is P-invariant, then we have operators $\mathrm{S}^{k}, \mathrm{D}^{k}: \mathcal{M}(\Omega) \rightarrow \mathcal{M}(\Omega)$. Remark 2.18. Obviously, Propositions 2.4 and 2.5 remain valid in this new context.

Proposition 2.19. Let $\Omega$ be an open set of the complex plane and $\gamma \simeq 0(\bmod \Omega)$. Then, given $f \in \mathcal{H}(\Omega)$, an integer $k \geq 0$ and $x \in \Omega^{(k)}$, we have

$$
\mathrm{D}^{k} f(x)=\left(\prod_{j=0}^{k-1} \frac{\lambda^{k-j}-\lambda^{j-k}}{\lambda-\lambda^{-1}}\right) \frac{1}{2 \pi i} \int_{\gamma} \frac{f(y)}{\Phi_{k+1}(x, y)} d y
$$

if $x$ is the base point of a $\mathrm{P}$-sequence $\left(x_{t}\right)$ with $\operatorname{Ind}\left(\gamma, x_{j-\frac{k}{2}}\right)=1$ for $j=0,1, \ldots, k$.

Proof. The cases $k=0$ and $k=1$ are Cauchy's integral formula and (7), respectively. The general case follows by induction from (7) and the relation

$$
\mathrm{D}\left[\frac{1}{\Phi_{k}(\cdot, y)}\right](x)=\frac{\lambda^{k}-\lambda^{-k}}{\lambda-\lambda^{-1}} \Phi_{k+1}(x, y)^{-1}
$$

which can be checked by the reader. This completes the proof.

Corollary 2.20. Let $\Omega$ be an open subset of the complex plane and $\gamma \simeq 0(\bmod \Omega)$. Then, given $f \in \mathcal{H}(\Omega)$, the expression

$$
\partial_{k} f(x)=\frac{1}{2 \pi i} \int_{\gamma} \frac{f(y)}{\Phi_{k+1}(x, y)} d y
$$

defines a linear operator $\partial_{k}: \mathcal{H}(\Omega) \rightarrow \mathcal{H}\left(\Omega^{(k)}\right)$. Moreover, we have

$$
\partial_{k} f(x)=\sum_{j=0}^{k} \operatorname{Res}_{\mid y=x_{j-\frac{k}{2}}}\left(\frac{f(y)}{\Phi_{k+1}(x, y)}\right) .
$$

Remark 2.21. It turns out that Lemma 2.11 is now a consequence of Corollary 2.20, Remark 2.22. When $x$ is the base point of a P-sequence $\left(x_{t}\right)$ with $x_{j-\frac{k}{2}}$ pairwise distinct for $j=0,1, \ldots, k$, we have by Corollary 2.20

$$
\partial_{k} f(x)=\sum_{j=0}^{k} \frac{f\left(x_{j-\frac{k}{2}}\right)}{\prod_{0 \leq i \neq j \leq k}\left(x_{j-\frac{k}{2}}-x_{i-\frac{k}{2}}\right)} .
$$

As we explain in Remark 3.11 this is useful to display our Rodrigues formulas given by Theorem 3.10. We recall that Nikiforov, Suslov and Uvarov give similar expressions for less intrinsic operators in [14, 3.2.3].

Remark 2.23. Under the hypothesis of Remark 2.22, Theorem 2.14 gives

$$
f(x)=\sum_{k=0}^{r}\left(\sum_{j=0}^{k} \frac{f\left(x_{j}\right)}{\prod_{i \neq j}\left(x_{j}-x_{i}\right)}\right) \prod_{j=0}^{k-1}\left(x-x_{j}\right)
$$

which is Newton's divided difference formula for the interpolation polynomial. In fact, as the referee of this paper has pointed out, the formula given in Remark 2.22 can be proved by induction. In particular, it is possible to obtain an alternative proof of Theorem 2.14 from the theory of interpolation polynomials. We prefer our proof since it shows how the polynomials $\Phi_{k}$ are related to D; see Lemma 2.10.

Remark 2.24. The operators $\partial_{k}: \mathcal{H}(\Omega) \rightarrow \mathcal{H}\left(\Omega^{(k)}\right)$ provide the natural framework to develop a generalization of Theorem 2.14. Namely, in a separate work 13, we study the convergence of the Taylor series associated to the operators $\partial_{k}$ of a given function $f \in \mathcal{H}(\Omega)$ and its relation with the basic hypergeometric functions. 


\section{TAYLOR COEFFICIENTS AND RODRIGUES FORMUlA}

In this section we introduce the corresponding hypergeometric operator. Then we compute the Taylor coefficients of its eigenfunctions. Also, we provide a general procedure to obtain Rodrigues type formulas. At the end of this section, we shall study the discrete orthogonal relations which arise from our techniques.

3.1. The hypergeometric operator. Given a symmetric polynomial $\mathrm{P}$ in the set $\mathcal{P}$, we consider the associated Askey-Wilson operator D and its companion S. Let $\sigma \in \mathbb{P}_{2}[x]$ and $\tau \in \mathbb{P}_{1}[x]$ given by

$$
\sigma(x)=\alpha_{2} x^{2}+\alpha_{1} x+\alpha_{0} \quad \text { and } \quad \tau(x)=\beta_{1} x+\beta_{0} .
$$

The aim of this section is to study the polynomic eigenfunctions of the following operator:

$$
\mathrm{L}=\sigma \mathrm{D}^{2}+\tau \mathrm{SD} .
$$

$\mathrm{L}$ will be called the hypergeometric operator associated to $\mathrm{P}, \sigma$ and $\tau$. In Section 4 we shall study in detail some relevant particular cases. It follows by Proposition 2.2 that $\mathrm{L}\left(\mathbb{P}_{k}[x]\right) \subset \mathbb{P}_{k}[x]$ for $k \geq 1$. Moreover, if $f(x)=x^{k}$ we obtain

$$
\mathrm{L} f(x)=-\mu_{k} x^{k}+\ldots,
$$

where the dots stand for terms of lower degree and

$$
\mu_{k}=-\frac{\lambda^{k}-\lambda^{-k}}{\left(\lambda-\lambda^{-1}\right)^{2}}\left[\left(\alpha_{2}+\frac{\lambda-\lambda^{-1}}{2} \beta_{1}\right) \lambda^{k-1}-\left(\alpha_{2}-\frac{\lambda-\lambda^{-1}}{2} \beta_{1}\right) \lambda^{1-k}\right] .
$$

As an endomorphism of $\mathbb{P}_{k}[x]$, the hypergeometric operator L depends continuously on $\mathrm{P}, \sigma$ and $\tau$. Therefore, taking suitable limits for $\lambda= \pm 1$, it turns out that $\mu_{k}$ is a continuous function on $\lambda$. The following formula, for which we should take limits in $\lambda$ when $\lambda= \pm 1$, will be useful in Section 4.

$$
\mu_{k}-\mu_{j}=\frac{\lambda^{j-k}-\lambda^{k-j}}{\left(\lambda-\lambda^{-1}\right)^{2}}\left[\left(\alpha_{2}+\frac{\lambda-\lambda^{-1}}{2} \beta_{1}\right) \lambda^{j+k-1}-\left(\alpha_{2}-\frac{\lambda-\lambda^{-1}}{2} \beta_{1}\right) \lambda^{1-j-k}\right] .
$$

Now we point out some relevant remarks on the operator L:

- If $\mathrm{L}^{\mathrm{P}}$ stands for the hypergeometric operator associated to the symmetric polynomial $\mathrm{P}$ and we consider some $g=(\eta, \zeta) \in \mathfrak{T}(\mathbb{C}) \rtimes G L(\mathbb{C})=\operatorname{Aff}(\mathbb{C})$, it follows easily from Remark 2.1 that

$$
g \mathrm{~L}^{\mathrm{P}} g^{-1}=\zeta^{2} \sigma^{g}\left(\mathrm{D}^{g \cdot \mathrm{P}}\right)^{2}+\zeta \tau^{g} \mathrm{~S}^{g \cdot \mathrm{P}} \mathrm{D}^{g \cdot \mathrm{P}},
$$

where $(g \cdot f)(x)=f\left(g^{-1}(x)\right), \sigma^{g}(x)=\sigma\left(g^{-1}(x)\right)$ and $\tau^{g}(x)=\tau\left(g^{-1}(x)\right)$.

- When $\left(x_{t}\right)$ is a P-sequence with $x_{t-\frac{1}{2}} \neq x_{t+\frac{1}{2}}$ for $t=0, \pm \frac{1}{2}$, we can write

$$
\begin{array}{r}
\mathrm{L} f\left(x_{0}\right)=\frac{\sigma\left(x_{0}\right)}{x_{1 / 2}-x_{-1 / 2}}\left[\frac{f\left(x_{1}\right)-f\left(x_{0}\right)}{x_{1}-x_{0}}-\frac{f\left(x_{0}\right)-f\left(x_{-1}\right)}{x_{0}-x_{-1}}\right] \\
+\frac{\tau\left(x_{0}\right)}{2}\left[\frac{f\left(x_{1}\right)-f\left(x_{0}\right)}{x_{1}-x_{0}}+\frac{f\left(x_{0}\right)-f\left(x_{-1}\right)}{x_{0}-x_{-1}}\right] .
\end{array}
$$

In fact, there exist a limiting version of this equality for any P-sequence $\left(x_{t}\right)$. Moreover, since $x_{1}-x_{-1}=2 a\left(x_{1 / 2}-x_{-1 / 2}\right)$ by Corollary 1.7, L $f\left(x_{0}\right)$ can be written in terms of $x_{t}$ with $t \in \mathbb{Z}$. In other words, since the subsequence of $\left(x_{t}\right)$ which arise when $t$ runs over $\mathbb{Z}$ is a $\mathrm{P}_{2}$-sequence with the terminology introduced after Lemma 2.8, we can say that $\mathrm{L}$ only depends on $\mathrm{P}_{2}$. 
- If the symmetric polynomial $\mathrm{P}$ is of type $\mathbf{O}$ (resp. E), it can be checked that $\mathrm{P}_{2}$ is of type $\mathbf{A}$ (resp. $\mathbf{C}$ ). Besides, if $\mathrm{P}$ is of type $\mathbf{A}$ (resp. $\mathbf{C}$ ), we also have that $\mathrm{P}_{2}$ is of type $\mathbf{A}$ (resp. $\mathbf{C}$ ). Therefore, taking into account the fact that $\mathrm{L}$ only depends on $\mathrm{P}_{2}$, we do not lose generality if we restrict our study to the canonical forms $\mathbf{T}, \mathbf{G}, \mathbf{Q}, \mathbf{A}$ and $\mathbf{C}$. This point of view leads to a different and somehow more natural classification than the one given in [14, 3.4]. Namely, while our classification is purely complex, it can be said that the classification given in [14] lives over the real field. As we point out below, another remarkable advantage of our formulation is that affine transformations become trivial.

- If $\lambda= \pm i$, we have $\mathrm{D}^{2}=\left(\lambda+\lambda^{-1}\right) \partial_{2}=0$. In particular, $\mathrm{L}=\tau \mathrm{SD}$ and so we get a degenerate case. This phenomenon can be avoided by considering the more general operator

$$
\mathrm{L}^{\prime}=\sigma \partial_{2}+\tau \mathrm{SD}
$$

It is not difficult to adapt the methods we shall develop to this case. When $\lambda= \pm i$ in $\mathrm{P}$, it can be checked that $\mathrm{P}_{2}$ is of type $\mathbf{E}$. Therefore, the even canonical form can be regarded from this alternative point of view. It can be shown that $\mathrm{L}^{\prime}$ is a polynomial function of $\sigma, \tau$ and $\mathrm{P}_{2}$ only.

- The polynomial $\mathrm{P}_{2}$ cannot be of type $\mathbf{O}$. However, we can construct a limiting operator which somehow corresponds to certain $\mathrm{P}_{2}$ of type $\mathbf{O}$. Namely, it suffices to take a sequence $\mathrm{P}(n)$ in $\mathcal{P}$ such that the sequence $\mathrm{P}(n)_{2}$ converges in $\mathcal{P}$ to a symmetric polynomial of type $\mathbf{O}$. It turns out that, in this situation, the sequence $\mathrm{P}(n)$ does not converge when $n \rightarrow$ $\infty$. However, it can be checked that the corresponding hypergeometric operators $\mathrm{L}(n)$ do converge. Therefore, the oscillating canonical form could be analyzed by means of the operator

$$
\mathrm{L}=\lim _{n \rightarrow \infty} \mathrm{L}(n) .
$$

Although it can be studied what happens with our methods after taking this limit, we shall not cover this problem here.

3.2. Taylor coefficients and Rodrigues formula. The first step in our process is the following commutation relation for the iterated hypergeometric operators. Expressions of the same kind can also be found in $[3$.

Lemma 3.1. Taking $\sigma_{0}=\sigma, \tau_{0}=\tau$ and given $j \geq 0$, let us define the polynomials $\sigma_{j} \in \mathbb{P}_{2}[x]$ and $\tau_{j} \in \mathbb{P}_{1}[x]$ inductively as follows:

$$
\begin{aligned}
\sigma_{j+1}(x) & =\mathrm{S} \sigma_{j}(x)+(a+1)(A(x)-x) \mathrm{S} \tau_{j}(x)+a \delta(x) \mathrm{D} \tau_{j}(x), \\
\tau_{j+1}(x) & =\mathrm{D} \sigma_{j}(x)+a \mathrm{~S} \tau_{j}(x)+(a+1)(A(x)-x) \mathrm{D} \tau_{j}(x) .
\end{aligned}
$$

Then, the iterated hypergeometric operator $\mathrm{L}_{j}=\sigma_{j} \mathrm{D}^{2}+\tau_{j} \mathrm{SD}$ satisfies

$$
\mathrm{D}\left(\mathrm{L}_{j}-\mu_{j}\right)=\left(\mathrm{L}_{j+1}-\mu_{j+1}\right) \mathrm{D} .
$$


Proof. Applying the Leibniz rules and the commuting relations developed for the operators S and D in Propositions 2.4 and 2.5, we easily obtain

$$
\begin{aligned}
\mathrm{DL}_{j} f(x) & =\mathrm{D}\left(\sigma_{j} \mathrm{D}^{2} f\right)(x)+\mathrm{D}\left(\tau_{j} \mathrm{SD} f\right)(x) \\
& =\mathrm{S} \sigma_{j}(x) \mathrm{D}^{3} f(x)+\mathrm{D} \sigma_{j}(x) \mathrm{SD}^{2} f(x)+\mathrm{S} \tau_{j}(x) \mathrm{DSD} f(x)+\mathrm{D} \tau_{j}(x) \mathrm{S}^{2} \mathrm{D} f(x) \\
& =\mathrm{S} \sigma_{j}(x) \mathrm{D}^{3} f(x)+\mathrm{D} \sigma_{j}(x) \mathrm{SD}^{2} f(x) \\
& +\mathrm{S} \tau_{j}(x)\left((a+1)(A(x)-x) \mathrm{D}^{3} f(x)+a \mathrm{SD}^{2} f(x)\right) \\
& +\mathrm{D} \tau_{j}(x)\left(a \delta(x) \mathrm{D}^{3} f(x)+(a+1)(A(x)-x) \mathrm{SD}^{2} f(x)+\mathrm{D} f(x)\right) \\
& =\sigma_{j+1}(x) \mathrm{D}^{3} f(x)+\tau_{j+1}(x) \mathrm{SD}^{2} f(x)+\mathrm{D} \tau_{j}(x) \mathrm{D} f(x) .
\end{aligned}
$$

In other words, $\mathrm{DL}_{j} f(x)=\left(\mathrm{L}_{j+1}+\mathrm{D} \tau_{j}\right) \mathrm{D} f(x)$, where $\mathrm{D} \tau_{j}$ is a constant $\nu_{j}$ since $\tau_{j} \in \mathbb{P}_{1}[x]$. On the other hand, taking $f(x)=x^{k}$, we define $\mu_{j, k}$ by the relation

$$
\mathrm{L}_{j} f(x)=-\mu_{j, k} x^{k}+\cdots,
$$

where the dots stand for terms of lower degree. Then, Proposition 2.2 gives

$$
-\left(\frac{\lambda^{k}-\lambda^{-k}}{\lambda-\lambda^{-1}}\right) \mu_{j, k}=\left(\frac{\lambda^{k}-\lambda^{-k}}{\lambda-\lambda^{-1}}\right)\left(-\mu_{j+1, k-1}+\nu_{j}\right) .
$$

By continuity, we have $\mu_{j+1, k-1}=\mu_{j, k}+\nu_{j}$ or equivalently

$$
\mu_{j, k}=\mu_{j+k}+\sum_{i=0}^{j-1} \nu_{i}
$$

In particular, we obtain that $\mu_{j}+\nu_{0}+\ldots+\nu_{j-1}=\mu_{j, 0}=0$. Therefore, we deduce the identity $\nu_{j}=\mu_{j}-\mu_{j+1}$ as we wanted. This completes the proof.

Given a symmetric polynomial $\mathrm{P}(x, y)=x^{2}+y^{2}-2 a x y-2 b(x+y)+c$, let us take a P-function $\theta_{t}$. Then we define the auxiliary functions

$$
\tilde{\sigma}_{j}^{ \pm}(t)=\sigma_{j}\left(\theta_{t}\right) \pm \frac{\theta_{t+\frac{1}{2}}-\theta_{t-\frac{1}{2}}}{2} \tau_{j}\left(\theta_{t}\right) \quad \text { for } \quad j \geq 0 .
$$

We shall write $\widetilde{\sigma}^{ \pm}$to denote the functions $\tilde{\sigma}_{0}^{ \pm}$, respectively.

Lemma 3.2. The auxiliary functions satisfy the following relations:

$$
\tilde{\sigma}_{j}^{+}(t)=\widetilde{\sigma}^{+}\left(t+\frac{j}{2}\right) \quad \text { and } \quad \tilde{\sigma}_{j}^{-}(t)=\widetilde{\sigma}^{-}\left(t-\frac{j}{2}\right) .
$$

Proof. By definition we have

$$
\begin{aligned}
\tilde{\sigma}_{j+1}^{ \pm}(t) & =\sigma_{j+1}\left(\theta_{t}\right) \pm \frac{1}{2}\left(\theta_{t+\frac{1}{2}}-\theta_{t-\frac{1}{2}}\right) \tau_{j+1}\left(\theta_{t}\right) \\
& =\mathrm{S} \sigma_{j}\left(\theta_{t}\right)+(a+1)\left(A\left(\theta_{t}\right)-\theta_{t}\right) \mathrm{S} \tau_{j}\left(\theta_{t}\right)+a \delta\left(\theta_{t}\right) \mathrm{D} \tau_{j}\left(\theta_{t}\right) \\
& \pm \frac{1}{2}\left(\theta_{t+\frac{1}{2}}-\theta_{t-\frac{1}{2}}\right)\left(\mathrm{D} \sigma_{j}\left(\theta_{t}\right)+a \mathrm{~S} \tau_{j}\left(\theta_{t}\right)+(a+1)\left(A\left(\theta_{t}\right)-\theta_{t}\right) \mathrm{D} \tau_{j}\left(\theta_{t}\right)\right) .
\end{aligned}
$$

Then, by Corollary 1.7 and (3), we get

$$
\begin{aligned}
\widetilde{\sigma}_{j+1}^{ \pm}(t) & =\frac{\sigma_{j}\left(\theta_{t+\frac{1}{2}}\right)+\sigma_{j}\left(\theta_{t-\frac{1}{2}}\right)}{2}+\frac{\theta_{t+1}-2 \theta_{t}+\theta_{t-1}}{8}\left(\tau_{j}\left(\theta_{t+\frac{1}{2}}\right)+\tau_{j}\left(\theta_{t-\frac{1}{2}}\right)\right) \\
& +\frac{\theta_{t+1}-\theta_{t-1}}{8}\left(\tau_{j}\left(\theta_{t+\frac{1}{2}}\right)-\tau_{j}\left(\theta_{t-\frac{1}{2}}\right)\right) \pm \frac{\theta_{t+1}-\theta_{t-1}}{8}\left(\tau_{j}\left(\theta_{t+\frac{1}{2}}\right)+\tau_{j}\left(\theta_{t-\frac{1}{2}}\right)\right) \\
& \pm \frac{\theta_{t+1}-2 \theta_{t}+\theta_{t-1}}{8}\left(\tau_{j}\left(\theta_{t+\frac{1}{2}}\right)-\tau_{j}\left(\theta_{t-\frac{1}{2}}\right)\right) \pm \frac{\sigma_{j}\left(\theta_{t+\frac{1}{2}}\right)-\sigma_{j}\left(\theta_{t-\frac{1}{2}}\right)}{2} .
\end{aligned}
$$

Simplifying the expression above, we obtain $\widetilde{\sigma}_{j+1}^{ \pm}(t)=\widetilde{\sigma}_{j}^{ \pm}\left(t \pm \frac{1}{2}\right)$. Therefore, the stated relations follow easily by induction and so the proof is completed. 
Now, given a positive integer $d$, we assume that $\mu_{j} \neq \mu_{k}$ for $0 \leq j<k \leq d$. In particular, this condition implies that $\mathrm{L}$ is a diagonalizable operator in $\mathbb{P}_{d}[x]$. Therefore, for any $0 \leq k \leq d$, there exists an eigenfunction $f_{k} \in \mathbb{P}_{k}[x]$ with degree $k$ satisfying

$$
\mathrm{L} f_{k}(x)+\mu_{k} f_{k}(x)=0 .
$$

Moreover, if we define $f_{k}^{(j)}=\mathrm{D}^{j} f_{k}$, then Lemma 3.1 and induction gives

$$
\mathrm{L}_{j} f_{k}^{(j)}+\left(\mu_{k}-\mu_{j}\right) f_{k}^{(j)}=0 .
$$

Lemma 3.3. The following relation holds for any $j \geq 0$

$$
\widetilde{\sigma}_{j}^{-}(t) f_{k}^{(j+2)}\left(\theta_{t}\right)+\tau_{j}\left(\theta_{t}\right) f_{k}^{(j+1)}\left(\theta_{t+\frac{1}{2}}\right)+\left(\mu_{k}-\mu_{j}\right) f_{k}^{(j)}\left(\theta_{t}\right)=0 .
$$

Proof. Applying the definition of $\tilde{\sigma}_{j}^{-}$in terms of $\sigma_{j}$ and $\tau_{j}$ and relation (4), we can rewrite the left-hand side as

$$
\text { LHS }=\sigma_{j}\left(\theta_{t}\right) \mathrm{D}^{2} f_{k}^{(j)}\left(\theta_{t}\right)+\tau_{j}\left(\theta_{t}\right) \mathrm{SD}_{k}^{(j)}\left(\theta_{t}\right)+\left(\mu_{k}-\mu_{j}\right) f_{k}^{(j)}\left(\theta_{t}\right) .
$$

In other words, the left-hand side coincides with $\mathrm{L}_{j} f_{k}^{(j)}\left(\theta_{t}\right)+\left(\mu_{k}-\mu_{j}\right) f_{k}^{(j)}\left(\theta_{t}\right)$, which vanishes by relation (8). Therefore, the proof is completed.

If we take the P-function $\theta_{t}$ in such a way that $\widetilde{\sigma}^{-}(0)=0$, then we can give explicit expressions for the eigenfunctions $f_{0}, f_{1}, \ldots, f_{d}$ in terms of the Taylor formula developed in Section 2 ,

Theorem 3.4. Let us assume that $\widetilde{\sigma}^{-}(0)=0$. Then the eigenfunctions $f_{0}, f_{1}, \ldots, f_{d}$ of $\mathrm{L}$ satisfy the following identities:

$$
f_{k}(x)=\partial_{k} f_{k} \sum_{j=0}^{k}\left(\prod_{i=j}^{k-1} \frac{\lambda^{1+i}-\lambda^{-1-i}}{\lambda-\lambda^{-1}} \frac{\tau_{i}\left(\theta_{i / 2}\right)}{\mu_{i}-\mu_{k}}\right) \prod_{i=0}^{j-1}\left(x-\theta_{i}\right),
$$

where $\partial_{k} f_{k}$ stands for the coefficient of $x^{k}$ in $f_{k}(x)$. Moreover, assuming $f_{k}\left(\theta_{0}\right) \neq 0$, we also have the following identities:

$$
f_{k}(x)=f_{k}\left(\theta_{0}\right) \sum_{j=0}^{k}\left(\prod_{i=0}^{j-1} \frac{\lambda-\lambda^{-1}}{\lambda^{1+i}-\lambda^{-1-i}} \frac{\mu_{i}-\mu_{k}}{\tau_{i}\left(\theta_{i / 2}\right)}\right) \prod_{i=0}^{j-1}\left(x-\theta_{i}\right) .
$$

Proof. Since $\widetilde{\sigma}^{-}(0)=0$, we obtain from Lemmas 3.2 and 3.3 the identity

$$
f_{k}^{(j)}\left(\theta_{j / 2}\right)=\frac{\tau_{j}\left(\theta_{j / 2}\right)}{\mu_{j}-\mu_{k}} f_{k}^{(j+1)}\left(\theta_{(j+1) / 2}\right) .
$$

If we write this recurrence in terms of the divided derivative operator, we get

$$
\partial_{j} f_{k}\left(\theta_{j / 2}\right)=\frac{\lambda^{1+j}-\lambda^{-1-j}}{\lambda-\lambda^{-1}} \frac{\tau_{j}\left(\theta_{j / 2}\right)}{\mu_{j}-\mu_{k}} \partial_{j+1} f_{k}\left(\theta_{(j+1) / 2}\right) .
$$

Then, the first relation follows by iterating this recurrence and applying Theorem 2.14. The second relation is an obvious consequence of the first relation and the identity

$$
f_{k}\left(\theta_{0}\right)=\partial_{k} f_{k} \prod_{i=0}^{k-1} \frac{\lambda^{1+i}-\lambda^{-1-i}}{\lambda-\lambda^{-1}} \frac{\tau_{i}\left(\theta_{i / 2}\right)}{\mu_{i}-\mu_{k}}
$$

which follows from the expression above for $j=0$. This completes the proof. 
Remark 3.5. Although using a less clear notation, Nikiforov, Suslov and Uvarov gave in [14,3.1.27] an equivalent identity to Lemma 3.2. Moreover, Lemma 3.3 is quite close to [14, 3.1.24]. However, since there is no Taylor formula for the Askey-Wilson operators in [14, it seems that the authors did not see the relevance of these results to obtain explicit polynomic expressions for the eigenfunctions.

Remark 3.6. Replacing $\theta_{t}$ by the P-function $\theta_{t}^{\prime}=\theta_{ \pm\left(t-t_{0}\right)}$, we can use Theorem 3.4 to obtain explicit expressions of the eigenfunctions $f_{k}$ for each $t_{0}$ satisfying either $\tilde{\sigma}^{+}\left(t_{0}\right)=0$ or $\widetilde{\sigma}^{-}\left(t_{0}\right)=0$. Such points $t_{0}$ are those for which $\theta_{t_{0}}$ is a root of the polynomial $\mathrm{Q}(x)=\sigma(x)^{2}-\delta(x) \tau(x)^{2}$. Since the degree of $\mathrm{Q}$ is less than or equal to 4 , we obtain in the generic cases four explicit expressions. We shall see in Section 4 that there are only two cases, with distinct eigenvalues, for which $\mathrm{Q}$ has no roots.

Remark 3.7. The assumption $\mu_{j} \neq \mu_{k}$ for $0 \leq j<k \leq d$ on the eigenvalues is too restrictive in Theorem 3.4. Namely, assuming that $\widetilde{\sigma}^{-}(0)=0$, it can be proved that $\mathrm{L}$ is diagonalizable in $\mathbb{P}_{d}[x]$ if and only if

$$
\prod_{i=j}^{k-1} \frac{\lambda^{1+i}-\lambda^{-1-i}}{\lambda-\lambda^{-1}} \tau_{i}\left(\theta_{i / 2}\right)=0
$$

whenever $\mu_{j}=\mu_{k}$ for some $0 \leq j<k \leq d$. In this case, the first identity of Theorem 3.4 holds summing from $j_{k}=1+\max \left\{j: \mu_{j}=\mu_{k}\right\}$. In any case, for the sake of clarity, we shall assume that the eigenvalues are pairwise distinct.

The first relation in Theorem 3.4 is the appropriate one when we normalize the eigenfunctions $f_{k}$ so that they become monic polynomials. The second relation in Theorem 3.4 will be very useful in Section 4, since it will allow us to express the eigenfunctions $f_{k}$ in terms of basic hypergeometric functions. The next result can be regarded as an algorithm to obtain Rodrigues-type formulas in this setting. We recall that the P-invariant domains were already introduced in Section 2.3 .

Lemma 3.8. Let $\rho$ and $\rho_{1}$ be meromorphic functions in $\Omega$ for some $\mathrm{P}$-invariant open set $\Omega$. Then, the following are equivalent:

(a) For any function $f \in \mathcal{M}(\Omega)$, we have

$$
\rho(x) \mathrm{L} f(x)=\mathrm{D}\left(\rho_{1} \mathrm{D} f\right)(x) .
$$

(b) The functions $\rho$ and $\rho_{1}$ satisfy the relations

$$
\rho(x) \sigma(x)=\mathrm{S} \rho_{1}(x) \quad \text { and } \quad \rho(x) \tau(x)=\mathrm{D} \rho_{1}(x) .
$$

(c) The functions $\rho$ and $\rho_{1}$ satisfy the relations

(c1) $\mathrm{D}(\rho \sigma)(x)=(a+1)(A(x)-x) \mathrm{D}(\rho \tau)(x)+a \mathrm{~S}(\rho \tau)(x)$.

(c2) $\rho_{1}(x)=\mathrm{S}(\rho \sigma)(x)-a \delta(x) \mathrm{D}(\rho \tau)(x)-(a+1)(A(x)-x) \mathrm{S}(\rho \tau)(x)$.

Moreover, if the $\mathrm{P}$-function $\theta_{t}$ is not 1-periodic, they are also equivalent to

(d) The functions $\rho$ and $\rho_{1}$ satisfy the relations

(d1) $\rho\left(\theta_{t-\frac{1}{2}}\right) \widetilde{\sigma}^{+}\left(t-\frac{1}{2}\right)=\rho\left(\theta_{t+\frac{1}{2}}\right) \widetilde{\sigma}^{-}\left(t+\frac{1}{2}\right)$.

(d2) $\rho_{1}\left(\theta_{t}\right)=\frac{1}{2}\left(\rho\left(\theta_{t-\frac{1}{2}}\right) \widetilde{\sigma}^{+}\left(t-\frac{1}{2}\right)+\rho\left(\theta_{t+\frac{1}{2}}\right) \widetilde{\sigma}^{-}\left(t+\frac{1}{2}\right)\right)$.

Besides, the functional equation (c1) (resp. (c2)) is equivalent to (d1) (resp. (d2)). Finally, if (a) holds, then $\rho_{1}$ satisfies (c1) and (d1) for the iterated operator $\mathrm{L}_{1}$. 
Proof. Properties (a) and (b) are equivalent since, by Proposition 2.4, we have

$$
\begin{aligned}
\rho(x) \mathrm{L} f(x) & =\rho(x) \sigma(x) \mathrm{D}^{2} f(x)+\rho(x) \tau(x) \operatorname{SD} f(x), \\
\mathrm{D}\left(\rho_{1} \mathrm{D} f\right)(x) & =\left(\mathrm{S} \rho_{1}\right)(x) \mathrm{D}^{2} f(x)+\left(\mathrm{D} \rho_{1}\right)(x) \operatorname{SD} f(x) .
\end{aligned}
$$

To see $(\mathrm{b} \Rightarrow \mathrm{c})$, we apply Proposition 2.5

$$
\begin{aligned}
\mathrm{D}(\rho \sigma)(x) & =(a+1)(A(x)-x) \mathrm{D}^{2} \rho_{1}+a \mathrm{SD} \rho_{1}(x), \\
\mathrm{S}(\rho \sigma)(x) & =a \delta(x) \mathrm{D}(\rho \tau)(x)+(a+1)(A(x)-x) \mathrm{S}(\rho \tau)(x)+\rho_{1}(x) .
\end{aligned}
$$

Let us denote by $\mathrm{L}(\mathrm{c} 1)$ (resp. $\mathrm{R}(\mathrm{c} 1)$ ) the left- (resp. right-) hand side of (c1). For the equivalence between (c1) and (d1), we use Corollary 1.7 and (3). Namely, arguing as in Proposition 2.5, we easily get

$$
(\mathrm{L}(\mathrm{c} 1)-\mathrm{R}(\mathrm{c} 1))\left(\theta_{t}\right)=\frac{\tilde{\sigma}^{-}\left(t+\frac{1}{2}\right) \rho\left(\theta_{t+\frac{1}{2}}\right)-\tilde{\sigma}^{+}\left(t-\frac{1}{2}\right) \rho\left(\theta_{t-\frac{1}{2}}\right)}{\theta_{t+\frac{1}{2}}-\theta_{t-\frac{1}{2}}} .
$$

The equivalence between (c2) and (d2) follows by similar arguments. Now, let us assume that property (d) holds. Then we will have

$$
\begin{aligned}
& \rho_{1}\left(\theta_{t+\frac{1}{2}}\right)=\rho\left(\theta_{t}\right) \widetilde{\sigma}^{+}(t)=\rho\left(\theta_{t}\right)\left(\sigma\left(\theta_{t}\right)+\frac{\theta_{t+\frac{1}{2}}-\theta_{t-\frac{1}{2}}}{2} \tau\left(\theta_{t}\right)\right), \\
& \rho_{1}\left(\theta_{t-\frac{1}{2}}\right)=\rho\left(\theta_{t}\right) \widetilde{\sigma}^{-}(t)=\rho\left(\theta_{t}\right)\left(\sigma\left(\theta_{t}\right)-\frac{\theta_{t+\frac{1}{2}}-\theta_{t-\frac{1}{2}}}{2} \tau\left(\theta_{t}\right)\right) .
\end{aligned}
$$

In particular, $\mathrm{S} \rho_{1}\left(\theta_{t}\right)=\rho\left(\theta_{t}\right) \sigma\left(\theta_{t}\right)$ and $\mathrm{D} \rho_{1}\left(\theta_{t}\right)=\rho\left(\theta_{t}\right) \tau\left(\theta_{t}\right)$. That is, we have seen $(\mathrm{d} \Rightarrow \mathrm{b})$. It remains to see that (a) implies that $\rho_{1}$ satisfies (d1) for $\mathrm{L}_{1}$. Since (a) implies (d), we have by Lemma 3.2

$$
\rho_{1}\left(\theta_{t-\frac{1}{2}}\right) \widetilde{\sigma}_{1}^{+}\left(t-\frac{1}{2}\right)=\rho\left(\theta_{t}\right) \widetilde{\sigma}^{-}(t) \widetilde{\sigma}^{+}(t)=\rho_{1}\left(\theta_{t+\frac{1}{2}}\right) \widetilde{\sigma}_{1}^{-}\left(t+\frac{1}{2}\right) .
$$

But this relation is precisely (d1) for $\mathrm{L}_{1}$. Therefore, the proof is concluded.

Remark 3.9. $(\mathrm{d} 1 \Rightarrow \mathrm{c} 1)$ is the only implication that fails for 1-periodic P-functions.

Theorem 3.10. Let $\rho \in \mathcal{M}(\Omega)$ for some $\mathrm{P}$-invariant open set $\Omega$ and assume that $\rho$ satisfies the functional equation

$$
\rho\left(\theta_{t}\right) \widetilde{\sigma}^{+}(t)=\rho\left(\theta_{t+1}\right) \widetilde{\sigma}^{-}(t+1) .
$$

Then there exists a family of functions $\rho_{j} \in \mathcal{M}(\Omega)$, with $j \geq 0$ and such that $\rho_{0}=\rho$, determined by any of the identities

$$
\begin{aligned}
& \rho_{j+1}\left(\theta_{t}\right)=\rho_{j}\left(\theta_{t-\frac{1}{2}}\right) \tilde{\sigma}^{+}\left(t+\frac{(j-1)}{2}\right), \\
& \rho_{j+1}\left(\theta_{t}\right)=\rho_{j}\left(\theta_{t+\frac{1}{2}}\right) \tilde{\sigma}^{-}\left(t-\frac{(j-1)}{2}\right) .
\end{aligned}
$$

Moreover, this family of functions provide the following Rodrigues formula for the eigenfunctions $f_{0}, f_{1}, \ldots, f_{d}$ of $\mathrm{L}$ :

$$
\left(\prod_{j=0}^{k-1}\left(\mu_{j}-\mu_{k}\right)\right) \rho(x) f_{k}(x)=\partial_{k} f_{k}\left(\prod_{j=0}^{k-1} \frac{\lambda^{k-j}-\lambda^{j-k}}{\lambda-\lambda^{-1}}\right) \mathrm{D}^{k} \rho_{k}(x) .
$$

Proof. The recurrence relations given above for $\rho_{j}$ follow from Lemmas 3.2 and 3.8 . Besides, by Lemma 3.8, we have $\rho_{j}(x) \mathrm{L}_{j} f(x)=\mathrm{D}\left(\rho_{j+1} \mathrm{D} f\right)(x)$ for any $f \in \mathcal{M}(\Omega)$. If we combine this relation with (8), we get

$$
\left(\mu_{j}-\mu_{k}\right) \rho_{j}(x) f_{k}^{(j)}(x)=\mathrm{D}\left(\rho_{j+1} f_{k}^{(j+1)}\right)(x) .
$$


Then we differentiate successively this expression to obtain

$$
\left(\prod_{j=0}^{k-1}\left(\mu_{j}-\mu_{k}\right)\right) \rho(x) f_{k}(x)=\mathrm{D}^{k}\left(\rho_{k} f_{k}^{(k)}\right)=\partial_{k} f_{k}\left(\prod_{j=0}^{k-1} \frac{\lambda^{k-j}-\lambda^{j-k}}{\lambda-\lambda^{-1}}\right) \mathrm{D}^{k} \rho_{k}(x),
$$

where we have applied Proposition 2.2. This completes the proof.

Remark 3.11. Let us recall that, assuming that $x$ is the base point of a P-sequence with $x_{j-\frac{k}{2}}$ pairwise distinct for $j=0,1, \ldots, k$ and with the aid of Remark 2.22, we can display Rodrigues formula as follows:

$$
\mathrm{D}^{k} \rho_{k}(x)=\left(\prod_{j=0}^{k-1} \frac{\lambda^{k-j}-\lambda^{j-k}}{\lambda-\lambda^{-1}}\right) \sum_{j=0}^{k} \frac{\rho_{k}\left(x_{j-\frac{k}{2}}\right)}{\prod_{i \neq j}\left(x_{j-\frac{k}{2}}-x_{i-\frac{k}{2}}\right)} .
$$

Remark 3.12. The recurrence relations for the functions $\rho_{j}$ given in Theorem 3.10 have the corresponding analogs in [14, 3.2.11 and Pg 64]. However, the authors of 14] work with the functions $\rho_{j}^{\text {NSU }}$ defined by

$$
\rho_{j}^{\mathrm{NSU}}(s)=\rho_{j}\left(\theta_{s+\frac{j}{2}}\right) .
$$

Hence, these functions cannot be easily regarded as functions of the variable $\theta_{s}$. One of our main contributions is that we show how to find meromorphic solutions $\rho$ and $\rho_{j}$ (with the exception of the form $\mathbf{C}$, see Section 4) in the variable $\theta_{s}$. To that aim, it is essential to regard these recurrence relations in its most intrinsic form as in (c2) of Lemma 3.8. This result has no analog in [14.

Remark 3.13. Another remarkable advantage of this alternative formulation is that affine transformations become trivial. A good example to illustrate this is Fischer's paper [7, where the author gets a Rodrigues formula for the $q$-Racah polynomials. Fischer combines results from [2] and [14] with some independent computations. However, from our point of view, these kinds of results become trivial consequences. Namely, it is very well known that the $q$-Racah polynomials $\mathrm{R}_{k}$ are related to the Askey-Wilson polynomials $\mathrm{P}_{k}$ via

$$
\mathrm{R}_{k}(\bar{x} ; \alpha, \beta, \gamma, \delta)=\mathrm{P}_{k}(x ; a, b, c, d),
$$

where $\bar{x}=2 \sqrt{q \gamma \delta} x$ and

$$
\begin{array}{ll}
a=\sqrt{q \gamma \delta}, & b=\alpha \sqrt{q / \gamma \delta}, \\
c=\beta \sqrt{\delta q / \gamma}, & d=\sqrt{\gamma q / \delta} .
\end{array}
$$

Therefore, if we are given a Rodrigues formula for the Askey-Wilson polynomials (see Section (4) and we want a Rodrigues formula for the $q$-Racah polynomials, it suffices to take $\bar{\rho}_{k}(\bar{x})=\rho_{k}(x)$ and substitute the operator $\mathrm{D}$, associated to the $\mathrm{P}_{k}$ 's, by the operator $\overline{\mathrm{D}}$ associated to the $\mathrm{R}_{k}$ 's.

Remark 3.14. The version of Theorem 3.10 for the continuous canonical form, for which every P-sequence is constant and hence 1-periodic, is very well known. In this case we have $\sigma_{j}(x)=\sigma(x)$ and $\tau_{j}(x)=\tau(x)+j \sigma^{\prime}(x)$. Moreover, the functional equations given in Theorem 3.10 must be replaced by

$$
\frac{d(\rho \sigma)}{d x}(x)=\rho(x) \tau(x) \quad \text { and } \quad \rho_{j}(x)=\rho(x) \sigma^{j}(x) .
$$

This also follows from Lemmas 3.1 and 3.8 . But a direct proof is quite simpler. 
3.3. Discrete orthogonality relations. In this paragraph we shall assume the existence of meromorphic functions $\rho$ and $\rho_{j}$ satisfying the hypothesis of Theorem 3.10 and we show how these functions can be used to obtain discrete orthogonality relations. Given $f, g \in \mathbb{P}[x]$, we define $h(u, v)$ to be the symmetric polynomial which coincides with

$$
\frac{f(u) g(v)-f(v) g(u)}{v-u}
$$

whenever $u \neq v$. Then, we define the Wronskian $\mathrm{W}: \mathbb{P}[x] \times \mathbb{P}[x] \rightarrow \mathbb{P}[x]$ to be the bilinear form

$$
\mathrm{W}(f, g)(x)=h(A(x)+\sqrt{\delta(x)}, A(x)-\sqrt{\delta(x)}) .
$$

Recall that $\mathrm{W}(f, g) \in \mathbb{P}[x]$ for any $f, g \in \mathbb{P}[x]$ since $h(u, v)$ is symmetric in $(u, v)$.

Lemma 3.15. We have $\rho(x)(f \mathrm{~L} g-g \mathrm{~L} f)(x)=\mathrm{D}\left(\rho_{1} \mathrm{~W}(f, g)\right)(x)$ for all $x \in \Omega$.

Proof. Given a point $x \in \Omega$, we know by Lemma 3.8 that

$$
\rho(x)(f \mathrm{~L} g-g \mathrm{~L} f)(x)=\left(f \mathrm{D}\left(\rho_{1} \mathrm{D} g\right)-g \mathrm{D}\left(\rho_{1} \mathrm{D} f\right)\right)(x) .
$$

On the other hand, let us consider a P-sequence with base point $x$. Then we can assume by a continuity argument that $x_{t+\frac{1}{2}} \neq x_{t-\frac{1}{2}}$ for $t=0, \pm \frac{1}{2}$. In that case, since $x=x_{0}$, we can rewrite the right-hand side as follows:

$$
\begin{aligned}
\mathrm{RHS} & =\frac{f\left(x_{0}\right)}{x_{1 / 2}-x_{-1 / 2}}\left[\rho_{1}\left(x_{1 / 2}\right) \frac{g\left(x_{1}\right)-g\left(x_{0}\right)}{x_{1}-x_{0}}-\rho_{1}\left(x_{-1 / 2}\right) \frac{g\left(x_{0}\right)-g\left(x_{-1}\right)}{x_{0}-x_{-1}}\right] \\
& -\frac{g\left(x_{0}\right)}{x_{1 / 2}-x_{-1 / 2}}\left[\rho_{1}\left(x_{1 / 2}\right) \frac{f\left(x_{1}\right)-f\left(x_{0}\right)}{x_{1}-x_{0}}-\rho_{1}\left(x_{-1 / 2}\right) \frac{f\left(x_{0}\right)-f\left(x_{-1}\right)}{x_{0}-x_{-1}}\right] .
\end{aligned}
$$

Since the terms in $f\left(x_{0}\right) g\left(x_{0}\right)$ cancel, we easily obtain the desired relation.

Theorem 3.16. Let us consider a $\mathrm{P}$-sequence $\left(x_{j}\right)$ and a non-negative integer $m$. Then, if $\rho$ is regular at $x_{0}, x_{1}, \ldots, x_{m}$ and $\rho_{1}\left(x_{-1 / 2}\right)=\rho_{1}\left(x_{m+\frac{1}{2}}\right)=0$, then the eigenfunctions $f_{0}, f_{1}, \ldots, f_{m}$ satisfy the following orthogonality relations:

$$
\left\langle f_{j}, f_{k}\right\rangle=\sum_{i=0}^{m} f_{j}\left(x_{i}\right) f_{k}\left(x_{i}\right) \rho\left(x_{i}\right)\left(x_{i+\frac{1}{2}}-x_{i-\frac{1}{2}}\right)=0 \quad \text { for } \quad j \neq k .
$$

Proof. Lemma 3.15 gives

$$
\begin{aligned}
\langle f, \mathrm{~L} g\rangle-\langle\mathrm{L} f, g\rangle & =\sum_{i=0}^{m} \mathrm{D}\left(\rho_{1} \mathrm{~W}(f, g)\right)\left(x_{i}\right)\left(x_{i+\frac{1}{2}}-x_{i-\frac{1}{2}}\right) \\
& =\sum_{i=0}^{m} \rho_{1}\left(x_{i+\frac{1}{2}}\right) \mathrm{W}(f, g)\left(x_{i+\frac{1}{2}}\right)-\rho_{1}\left(x_{i-\frac{1}{2}}\right) \mathrm{W}(f, g)\left(x_{i-\frac{1}{2}}\right) \\
& =\left.\rho_{1}(x) \mathrm{W}(f, g)(x)\right|_{x=x_{-\frac{1}{2}}} ^{x=x_{m+\frac{1}{2}}}=0 .
\end{aligned}
$$

Taking $f=f_{j}, g=f_{k}$ and recalling that $\mu_{j} \neq \mu_{k}$ for $j \neq k$, the result follows.

Remark 3.17. The inner product described in Theorem 3.16 can be complex.

Remark 3.18. In Section 4 we shall study how free we are at the time of choosing the functions $\rho$ and $\rho_{j}$. Using this it can be checked that, when

$$
\operatorname{ord}_{x_{-1 / 2}}\left(\rho_{1}\right)>\operatorname{ord}_{x_{0}}\left(\rho_{1}\right)=\operatorname{ord}_{x_{1}}\left(\rho_{1}\right)=\cdots=\operatorname{ord}_{x_{m}}\left(\rho_{1}\right)<\operatorname{ord}_{x_{m+1 / 2}}\left(\rho_{1}\right),
$$

we can modify the election of $\rho$ and $\rho_{1}$ so that hypotheses of Theorem 3.16 hold. 


\section{EXPLICIT FORMULAS FOR THE CANONICAL FORMS}

In this section we apply our methods to study the hypergeometric polynomials that arise when $\mathrm{P}$ takes one of the following canonical forms: $\mathbf{T}, \mathbf{G}, \mathbf{Q}, \mathbf{A}$ or $\mathbf{C}$. Although the canonical forms $\mathbf{O}$ and $\mathbf{E}$ could also be treated with our methods, we shall not cover them here, as we explained at the beginning of Section 3 . In each case we shall follow the following steps:

1. Parameterization of $\sigma$ and $\tau$ in terms of certain (Laurent) polynomial(s).

2. Expressions for $\alpha_{2}, \beta_{1}, \sigma_{j}, \tau_{j}$ and $\mu_{k}$ in terms of our parameterization.

3. Taylor formulas for the eigenfunctions $f_{0}, f_{1}, \ldots, f_{d}$.

4. Rodrigues formulas for the eigenfunctions $f_{0}, f_{1}, \ldots, f_{d}$.

5. Classical families of hypergeometric polynomials which belong to that form.

When dealing with Rodrigues formulas, we shall give in each case a method to construct the function $\rho$. The iterated functions $\rho_{j}$ can be easily obtained from $\rho$ by applying Theorem 3.10 . On the other hand, we shall assume again in what follows that the eigenvalues $\mu_{k}$ are pairwise distinct.

4.1. Continuous case. $\mathrm{P}(x, y)=x^{2}+y^{2}-2 x y$ and $\theta_{t}=x_{0}$.

1. In this case we do not need any parameterization.

2. It can be checked that we have

$$
\begin{gathered}
\mu_{k}-\mu_{i}=(i-k)\left(\alpha_{2}(i+k)-\alpha_{2}+\beta_{1}\right), \\
\sigma_{j}(x)=\sigma(x), \quad \tau_{j}(x)=\tau(x)+j \sigma^{\prime}(x) .
\end{gathered}
$$

3. Taking $x_{0}$ so that $\sigma\left(x_{0}\right)=0$, we obtain from Theorem 3.4

$$
\partial_{j} f_{k}\left(x_{0}\right)=\partial_{k} f_{k}\left(\begin{array}{l}
k \\
j
\end{array}\right) \prod_{i=j}^{k-1} \frac{\tau\left(x_{0}\right)+i \sigma^{\prime}\left(x_{0}\right)}{\alpha_{2}(k+i)-\alpha_{2}+\beta_{1}} .
$$

4. As we have pointed out in Remark 3.14, the Rodrigues formulas for this case are given by the solutions of the functional equations

$$
\frac{d(\rho \sigma)}{d x}(x)=\rho(x) \tau(x) \quad \text { and } \quad \rho_{j}(x)=\rho(x) \sigma^{j}(x) .
$$

In general, we cannot avoid obtaining ramified solutions. Quite surprisingly, this is the unique canonical form satisfying this property. Namely, as we shall see below, for any other canonical form we can always find non-ramified solutions of the corresponding functional equation for $\rho$.

5. Taking $\sigma(x)=x^{2}-1$ (resp. $\sigma(x)=x$ or $\sigma(x)=1$ ), we obtain the Jacobi (resp. Laguerre or Hermite) polynomials. Note that, if $\sigma(x)=1$ for all $x \in \mathbb{C}$, there are no roots of $\sigma$. In other words, the Hermite polynomials do not arise from the expressions given above and other methods are required to obtain them. However, the corresponding Rodrigues formula provided by Theorem 3.10 and Remark 3.14 also holds in this case. As we shall see in our analysis for the $\mathbf{T}$ canonical form, the $q$-Hermite polynomials behave in the same fashion.

4.2. Arithmetic case. $\mathrm{P}(x, y)=\left(x-y+\frac{1}{2}\right)\left(x-y-\frac{1}{2}\right)$ and $\theta_{t}=x_{0} \pm t$.

1. Let us consider two polynomials $\chi^{ \pm}(x)=\gamma_{2} x^{2}+\gamma_{1}^{ \pm} x+\gamma_{0}^{ \pm}$with the same coefficient for $x^{2}$. Clearly, there exists a bijective correspondence between these 
kinds of pairs and $\mathbb{P}_{2}[x] \times \mathbb{P}_{1}[x]$ given by

$$
\begin{array}{ll}
\chi^{+}(x)=\sigma(x)+\frac{\tau(x)}{2}, & \sigma(x)=\frac{\chi^{+}(x)+\chi^{-}(x)}{2}, \\
\chi^{-}(x)=\sigma(x)-\frac{\tau(x)}{2}, & \tau(x)=\chi^{+}(x)-\chi^{-}(x) .
\end{array}
$$

2. We have $\mu_{k}-\mu_{i}=(i-k)\left(\gamma_{2}(i+k)+\gamma_{1}^{+}-\gamma_{1}^{-}-\gamma_{2}\right), \alpha_{2}=\gamma_{2}$ and $\beta_{1}=\gamma_{1}^{+}-\gamma_{1}^{-}$. Taking the P-function $\theta_{t}=t$, we easily obtain the relations $\widetilde{\sigma}^{ \pm}(t)=\chi^{ \pm}(t)$. Thus, it turns out by Lemma 3.2 that $\tilde{\sigma}_{j}^{ \pm}(t)=\chi^{ \pm}\left(t \pm \frac{j}{2}\right)$. Therefore, $\sigma_{j}$ and $\tau_{j}$ are parameterized by

$$
\chi_{j}^{ \pm}(x)=\chi^{ \pm}\left(x \pm \frac{j}{2}\right) .
$$

In particular, we can write $\tau_{j}(x)=\chi^{+}\left(x+\frac{j}{2}\right)-\chi^{-}\left(x-\frac{j}{2}\right)$.

3. We have to consider two essentially different kinds of P-functions:

(a) If $\theta_{t}=x_{0}+t$, we have $\widetilde{\sigma}^{-}(t)=\chi^{-}\left(x_{0}+t\right)$. Thus, we take $x_{0}$ such that $\chi^{-}\left(x_{0}\right)=0$ to satisfy the hypothesis of Theorem 3.4. In that case, $\tau_{i}\left(\theta_{i / 2}\right)=\chi^{+}\left(x_{0}+i\right)$ and Theorem 3.4 gives

$$
\partial_{j} f_{k}\left(\theta_{j / 2}\right)=\partial_{k} f_{k}\left(\begin{array}{c}
k \\
j
\end{array}\right) \prod_{i=j}^{k-1} \frac{\chi^{+}\left(x_{0}+i\right)}{\gamma_{2}(i+k)+\gamma_{1}^{+}-\gamma_{1}^{-}-\gamma_{2}} .
$$

(b) If $\theta_{t}=x_{0}-t$, we have $\widetilde{\sigma}^{-}(t)=\chi^{+}\left(x_{0}-t\right)$. Thus, if we choose the point $x_{0}$ so that $\chi^{+}\left(x_{0}\right)=0$, we obtain $\tau_{i}\left(\theta_{i / 2}\right)=-\chi^{-}\left(x_{0}-i\right)$. In particular,

$$
\partial_{j} f_{k}\left(\theta_{j / 2}\right)=\partial_{k} f_{k}\left(\begin{array}{c}
k \\
j
\end{array}\right) \prod_{i=j}^{k-1} \frac{-\chi^{-}\left(x_{0}-i\right)}{\gamma_{2}(i+k)+\gamma_{1}^{+}-\gamma_{1}^{-}-\gamma_{2}} .
$$

Every case with pairwise distinct eigenvalues gives rise to an expression of this kind.

4. Taking the P-function $\theta_{t}=t$, the Rodrigues formulas given by Theorem 3.10 arise from the solutions of

$$
\begin{aligned}
\rho(t) \chi^{+}(t) & =\rho(t+1) \chi^{-}(t+1), \\
\rho_{j+1}(t) & =\rho_{j}\left(t \mp \frac{1}{2}\right) \chi^{ \pm}\left(t \pm \frac{j-1}{2}\right) .
\end{aligned}
$$

Non-vanishing entire solutions are available whenever $\chi^{ \pm}$are not identically zero. Moreover, those solutions can be obtained by multiplying the suitable solutions of the following root cases:

$$
\begin{aligned}
& \left.\left.\begin{array}{l|l}
\chi^{+}(x)=1 \\
\chi^{-}(x)=x-\xi^{-}
\end{array}\right\} \quad \rho(x)=\frac{1}{\Gamma\left(1+x-\xi^{-}\right)} \quad \begin{array}{l}
\chi^{+}(x)=\zeta \neq 0 \\
\chi^{-}(x)=1
\end{array}\right\} \quad \rho(x)=\zeta^{x}, \\
& \left.\left.\begin{array}{l|l}
\chi^{+}(x)=x-\xi^{+} \\
\chi^{-}(x)=1
\end{array}\right\} \quad \rho(x)=\frac{1}{\Gamma\left(1-x+\xi^{+}\right)} \quad \begin{array}{l}
\chi^{+}(x)=1 \\
\chi^{-}(x)=\zeta \neq 0
\end{array}\right\} \quad \rho(x)=\zeta^{-x} .
\end{aligned}
$$

Other solutions are available multiplying $\rho$ by a 1-periodic meromorphic function.

5. Taking $\chi^{ \pm}(x)=\left(x-\xi_{0}^{ \pm}\right)\left(x-\xi_{1}^{ \pm}\right)$, we obtain the Hahn polynomials
(a) $f_{k}(x)=f_{k}\left(\xi_{0}^{-}\right){ }_{3} F_{2}\left(\begin{array}{c}-k, \xi_{0}^{-}-x, k-1+\xi_{0}^{-}+\xi_{1}^{-}-\xi_{0}^{+}-\xi_{1}^{+} \\ \xi_{0}^{-}-\xi_{0}^{+}, \xi_{0}^{-}-\xi_{1}^{+}\end{array}\right)$,
(b) $f_{k}(x)=f_{k}\left(\xi_{0}^{+}\right){ }_{3} F_{2}\left(\begin{array}{c}-k, x-\xi_{0}^{+}, k-1+\xi_{0}^{-}+\xi_{1}^{-}-\xi_{0}^{+}-\xi_{1}^{+} \\ \xi_{0}^{-}-\xi_{0}^{+}, \xi_{1}^{-}-\xi_{0}^{+}\end{array} \mid 1\right)$. 
4.3. Quadratic case. $\mathrm{P}(x, y)=x^{2}+y^{2}-2 x y-\frac{1}{2}(x+y)+\frac{1}{16}$ and $\theta_{t}=\left(t+t_{0}\right)^{2}$.

1. Now we consider a polynomial $\chi(t)=\gamma_{4} t^{4}+\gamma_{3} t^{3}+\gamma_{2} t^{2}+\gamma_{1} t+\gamma_{0}$. Then, the parameterization is given by

$$
\begin{gathered}
\chi(t)=\sigma\left(t^{2}\right)+t \tau\left(t^{2}\right) \\
\sigma\left(t^{2}\right)=\frac{\chi(t)+\chi(-t)}{2} \quad \text { and } \quad \tau\left(t^{2}\right)=\frac{\chi(t)-\chi(-t)}{2 t} .
\end{gathered}
$$

2. We have $\alpha_{2}=\gamma_{4}, \beta_{1}=\gamma_{3}$ and

$$
\mu_{k}-\mu_{i}=(i-k)\left(\gamma_{4}(i+k)-\gamma_{4}+\gamma_{3}\right) .
$$

Taking $\theta_{t}=t^{2}$ and applying Lemma 3.2 , we get $\widetilde{\sigma}_{j}^{ \pm}(t)=\chi\left( \pm t+\frac{j}{2}\right)$ for $j \geq 0$. Hence, $\sigma_{j}$ and $\tau_{j}$ are parameterized by the polynomial

$$
\chi_{j}(x)=\chi\left(x+\frac{j}{2}\right) .
$$

In particular, we have

$$
\tau_{j}\left(t^{2}\right)=\frac{\chi\left(t+\frac{j}{2}\right)-\chi\left(-t+\frac{j}{2}\right)}{2 t} .
$$

3. It suffices to consider the P-functions $\theta_{t}=\left(t+t_{0}\right)^{2}$. In that case, we have $\tilde{\sigma}^{-}(t)=\chi\left(-t-t_{0}\right)$ and so we impose $\chi\left(-t_{0}\right)=0$. This condition provides the factorization $\chi(t)=\left(t+t_{0}\right) \chi_{0}(t)$ for some $\chi_{0} \in \mathbb{P}_{3}[x]$. Therefore, it turns out that $\tau_{i}\left(\theta_{i / 2}\right)=\chi_{0}\left(t_{0}+i\right)$. Theorem 3.4 gives

$$
\partial_{j} f_{k}\left(\theta_{j / 2}\right)=\partial_{k} f_{k}\left(\begin{array}{c}
k \\
j
\end{array}\right) \prod_{i=j}^{k-1} \frac{\chi_{0}\left(t_{0}+i\right)}{\gamma_{4}(i+k)-\gamma_{4}+\gamma_{3}} .
$$

Every case with pairwise distinct eigenvalues gives rise to an expression of this kind.

4. Taking the P-function $\theta_{t}=t^{2}$ and following Theorem 3.10, we have to solve

$$
\begin{aligned}
\rho\left(t^{2}\right) \chi(t) & =\rho\left((t+1)^{2}\right) \chi(-t-1), \\
\rho_{j+1}\left(t^{2}\right) & =\rho_{j}\left(\left(t \mp \frac{1}{2}\right)^{2}\right) \chi\left( \pm t+\frac{j-1}{2}\right) .
\end{aligned}
$$

Non-vanishing entire solutions are always available. Namely, any solution arises as a product of functions like

$$
\rho\left(t^{2}\right)=\frac{1}{\Gamma(1-\xi+t) \Gamma(1-\xi-t)},
$$

which solves the functional equation for $\chi(t)=t+\xi$ and $\xi \in \mathbb{C}$. Moreover, other solutions appear when we multiply $\rho$ by any even 1-periodic meromorphic function.

5. Taking $\chi(x)=\prod_{\nu=0}^{3}\left(x+\xi_{\nu}\right)$ and $t_{0}=\xi_{0}$, we obtain the Wilson polynomials

$$
f_{k}\left(t^{2}\right)=f_{k}\left(\xi_{0}^{2}\right){ }_{4} F_{3}\left(\begin{array}{c}
-k, \xi_{0}+t, \xi_{0}-t, k-1+\xi_{0}+\xi_{1}+\xi_{2}+\xi_{3} \\
\xi_{0}+\xi_{1}, \xi_{0}+\xi_{2}, \xi_{0}+\xi_{3}
\end{array} \mid 1\right)
$$


4.4. Some remarks on a functional equation. Finally, it remains to study the geometric and trigonometric canonical forms. In order to give explicit Rodrigues formulas for these particular cases, we shall need to solve functional equations of the following type:

$$
\mathrm{F}(x)=\mathrm{R}(x) \mathrm{F}(q x)
$$

where $\mathrm{R}$ stands for a non-zero rational function. An open subset $\Omega$ of the complex plane will be called $q$-invariant when $q \Omega=\Omega$. We are interested in non-zero meromorphic solutions defined in a $q$-invariant connected open set $\Omega$. We shall need to apply the following basic results.

Lemma 4.1. Let $\mathrm{F}_{1}$ and $\mathrm{F}_{2}$ be non-zero meromorphic solutions of the functional equation (9) in a q-invariant domain $\Omega$ with respect to the rational functions $\mathrm{R}_{1}$ and $\mathrm{R}_{2}$, respectively. Then $\mathrm{F}_{1} \mathrm{~F}_{2}$ and $\mathrm{F}_{1} / \mathrm{F}_{2}$ are non-zero meromorphic solutions of (9) in $\Omega$ with respect to the rational functions $\mathrm{R}_{1} \mathrm{R}_{2}$ and $\mathrm{R}_{1} / \mathrm{R}_{2}$, respectively.

Lemma 4.2. A function $\mathrm{F}$ solves (9) in a $q$-invariant domain $\Omega$ for some $q \in \mathbb{C}$ if and only if $\mathrm{F}$ solves the functional equation

$$
\mathrm{F}(x)=\mathrm{S}(x) \mathrm{F}\left(q^{-1} x\right) \quad \text { in } \Omega \text { with } \quad \mathrm{S}(x)=\frac{1}{\mathrm{R}\left(q^{-1} x\right)} .
$$

Lemma 4.3. Let us suppose that a given q-invariant connected open set $\Omega$ contains the point 0 . Then, if there exist a non-zero meromorphic solution of (9) in $\Omega$, it is unique up to a constant factor.

Proof. The quotient $\mathrm{F}$ of any two meromorphic solutions of the functional equation (9) satisfies $\mathrm{F}(x)=\mathrm{F}(q x)$ in $\Omega$. Therefore, it suffices to write this relation in terms of the Laurent series of $\mathrm{F}$ in a neighborhood of 0 . This completes the proof.

By Lemma 4.2, the case $|q|>1$ can be reduced to the case $|q|<1$. Now we provide some particular solutions for $|q|<1$ which will be useful in the general case. Namely, the $q$-shifted factorials

$$
\begin{array}{llll}
g_{\xi}(x)=(\xi x ; q)_{\infty} & \text { with } & \mathrm{R}(x)=1-\xi x & \text { and } \quad \Omega=\mathbb{C}, \\
h_{\xi}(x)=(q / \xi x ; q)_{\infty} & \text { with } & \mathrm{R}(x)=\frac{-\xi x}{1-\xi x} & \text { and } \quad \Omega=\mathbb{C} \backslash\{0\} .
\end{array}
$$

Given a non-zero rational function $\mathrm{R}$, we can always write $\mathrm{R}$ in the form

$$
\mathrm{R}(x)=\zeta x^{r} \prod_{k=1}^{n} \frac{1-\xi_{k} x}{1-\eta_{k} x}
$$

with $r \in \mathbb{Z}, \zeta$ a non-zero complex number and $\xi_{k}, \eta_{k} \in \mathbb{C}$ for $1 \leq k \leq n$. Then, using the particular solutions $g_{\xi}$ and $h_{\xi}$, Lemma 4.1 assures that the function

$$
\mathrm{F}=\left[\frac{g_{\gamma} h_{\gamma}}{g_{1} h_{1}}\right]\left(g_{1} h_{1}\right)^{r} \prod_{k=1}^{n} g_{\xi_{k}} / g_{\eta_{k}} \quad \text { with } \quad \gamma=(-1)^{r} \zeta
$$

solves the corresponding functional equation. $\mathrm{F}$ is a meromorphic function in $\mathbb{C} \backslash\{0\}$. In fact, if $\zeta=1$ and $r=0, \mathrm{~F}$ is meromorphic in $\mathbb{C}$ and we know by Lemma4.3 that $\mathrm{F}$ is the unique meromorphic solution in $\mathbb{C}$ up to a constant factor.

Remark 4.4. Let $\mathrm{G}$ be meromorphic in $\mathbb{C}$ and biperiodic with periods $2 \pi i$ and $\log q$. The function $\mathrm{F}$ defined by $\mathrm{F}(\exp y)=\mathrm{G}(y)$ is meromorphic in $\mathbb{C} \backslash\{0\}$ and solves the functional equation $\mathrm{F}(x)=\mathrm{F}(q x)$. The theory of elliptic functions 
provides infinitely many functions of this kind. In particular, this shows there is no uniqueness of meromorphic solutions in $\mathbb{C} \backslash\{0\}$ of the functional equation (9).

Remark 4.5. By the previous discussion, when $q^{n}=\prod_{k=1}^{n}\left(\xi_{k} / \eta_{k}\right)$ the function

$$
\mathrm{E}(z)=x^{n} \prod_{k=1}^{n} \frac{g_{\xi_{k}}(x) h_{\xi_{k}}(x)}{g_{\eta_{k}}(x) h_{\eta_{k}}(x)} \quad\left(x=e^{z}\right)
$$

is elliptic with periods $2 \pi i$ and $\log q$. Moreover, $\mathrm{E}$ has a zero at $-\log \xi_{k}$ and has a pole at $-\log \eta_{k}$ for $1 \leq k \leq n$. The rest of the zeros and poles of $\mathrm{E}$ can be obtained by periodicity. Therefore we have obtained, in our terminology, a proof of a classical existence result. This explicit expression allows us to explore the consequences derived from the non-uniqueness of solutions $\rho$ and $\rho_{j}$ of the Rodrigues formulas. We shall give more details on this topic in the following Sections 4.5 and 4.6.

Remark 4.6. The functional equation (9) has non-vanishing rational solutions for $q=e^{2 \pi i / n}$ whenever the following extra hypothesis holds:

$$
\prod_{k=0}^{n-1} \mathrm{R}\left(q^{k} x\right)=1
$$

These solutions could be used to obtain Rodrigues formulas for such values of $q$. However, we have decided not to include them in this paper for lack of space.

Remark 4.7. Putting $f(t)=\mathrm{F}\left(q^{t}\right)$, (9) takes the form $f(t)=\mathrm{R}\left(q^{t}\right) f(t+1)$. These kinds of equations have been solved in [14] for $|q|<1$ in terms of the $\Gamma_{q}$-function. In general, these solutions cannot be regarded as non-ramified solutions in $q^{t}$. This alternative functional equation is also considered in [15, where some solutions $f(t)=\mathrm{F}\left(q^{t}\right)$ are given. However, the authors seem not to be specially interested on solutions which are functions of the variable $q^{t}$ and they do not observe the role of elliptic functions here. For more on the connection between $q$-calculus and elliptic function theory, we refer the reader to the references [1] and [6].

4.5. Geometric case. $\mathrm{P}(x, y)=x^{2}+y^{2}-2 a x y$ and $\theta_{t}=q^{ \pm t} x_{0}$.

1. Let us consider two polynomials $\chi^{ \pm}(x)=\gamma_{2}^{ \pm} x^{2}+\gamma_{1}^{ \pm} x+\gamma_{0}$ with the same value at $x=0$. Then, the parameterization is given by

$$
\begin{array}{ll}
\chi^{+}(x)=\sigma(x)+\frac{\lambda-\lambda^{-1}}{2} x \tau(x), & \sigma(x)=\frac{\chi^{+}(x)+\chi^{-}(x)}{2}, \\
\chi^{-}(x)=\sigma(x)-\frac{\lambda-\lambda^{-1}}{2} x \tau(x), & \tau(x)=\frac{\chi^{+}(x)-\chi^{-}(x)}{\left(\lambda-\lambda^{-1}\right) x} .
\end{array}
$$

2. We have $\alpha_{2}=\left(\gamma_{2}^{+}+\gamma_{2}^{-}\right) / 2, \beta_{1}=\left(\gamma_{2}^{+}-\gamma_{2}^{-}\right) /\left(\lambda-\lambda^{-1}\right)$ and

$$
\mu_{k}-\mu_{i}=\frac{\lambda^{i-k}-\lambda^{k-i}}{\left(\lambda-\lambda^{-1}\right)^{2}}\left(\gamma_{2}^{+} \lambda^{i+k-1}-\gamma_{2}^{-} \lambda^{1-i-k}\right) .
$$

Taking $\theta_{t}=q^{t}$, it turns out that $\widetilde{\sigma}^{ \pm}(t)=\chi^{ \pm}\left(q^{t}\right)$ and $\widetilde{\sigma}_{j}^{ \pm}(t)=\chi^{ \pm}\left(\lambda^{ \pm j} q^{t}\right)$. In other words, the polynomials $\sigma_{j}$ and $\tau_{j}$ are parameterized by

$$
\chi_{j}^{ \pm}(x)=\chi^{ \pm}\left(\lambda^{ \pm j} x\right) .
$$


In particular, we obtain

$$
\tau_{j}(x)=\frac{\chi^{+}\left(\lambda^{j} x\right)-\chi^{-}\left(\lambda^{-j} x\right)}{\left(\lambda-\lambda^{-1}\right) x} .
$$

3. We have to consider two essentially different kind of P-functions:

(a) If $\theta_{t}=q^{t} x_{0}$, we have $\widetilde{\sigma}^{-}(t)=\chi^{-}\left(q^{t} x_{0}\right)$. Thus, we require $\chi^{-}\left(x_{0}\right)=0$ to satisfy the hypothesis of Theorem 3.4. Under this assumption, we have $\tau_{i}\left(\theta_{i / 2}\right)=\chi^{+}\left(q^{i} x_{0}\right) /\left(\lambda-\lambda^{-1}\right) \lambda^{i} x_{0}$ and Theorem 3.4 gives

$$
\partial_{j} f_{k}\left(\theta_{j / 2}\right)=\partial_{k} f_{k}\left[\begin{array}{c}
k \\
j
\end{array}\right]_{q} q^{-2\left[\left(\begin{array}{c}
k \\
2
\end{array}\right)-\left(\begin{array}{c}
j \\
2
\end{array}\right)\right]} \prod_{i=j}^{k-1} \frac{\chi^{+}\left(q^{i} x_{0}\right) / x_{0}}{\gamma_{2}^{+}-\gamma_{2}^{-} q^{1-i-k}} .
$$

(b) If $\theta_{t}=q^{-t} x_{0}$, we have $\widetilde{\sigma}^{-}(t)=\chi^{+}\left(q^{-t} x_{0}\right)$. Thus, we take $x_{0}$ such that $\chi^{+}\left(x_{0}\right)=0$. This gives $\tau_{i}\left(\theta_{i / 2}\right)=-\lambda^{i} \chi^{-}\left(q^{-i} x_{0}\right) /\left(\lambda-\lambda^{-1}\right) x_{0}$. In particular,

$$
\partial_{j} f_{k}\left(\theta_{j / 2}\right)=\partial_{k} f_{k}\left[\begin{array}{c}
k \\
j
\end{array}\right]_{q} q^{-\left[\left(\begin{array}{c}
k \\
2
\end{array}\right)-\left(\begin{array}{c}
j \\
2
\end{array}\right)\right]} \prod_{i=j}^{k-1} \frac{-\chi^{-}\left(q^{-i} x_{0}\right) / x_{0}}{\gamma_{2}^{+}-\gamma_{2}^{-} q^{1-i-k}} .
$$

Every case with pairwise distinct eigenvalues gives rise to an expression of this kind. When $x_{0}=0$, the terms $\chi^{ \pm}\left(q^{ \pm i} x_{0}\right) / x_{0}$ must be replaced by the obvious limits.

4. Taking the P-function $\theta_{t}=q^{t}$ and following Theorem 3.10, we have to obtain non-zero meromorphic solutions of

$$
\begin{aligned}
\rho(x) \chi^{+}(x) & =\rho(q x) \chi^{-}(q x), \\
\rho_{j+1}(x) & =\rho_{j}\left(\lambda^{\mp 1} x\right) \chi^{ \pm}\left(\lambda^{ \pm(j-1)} x\right) .
\end{aligned}
$$

in a P-invariant domain $\Omega$. Non-vanishing meromorphic solutions in $\mathbb{C} \backslash\{0\}$ are available whenever $|q|<1$ and $\chi^{ \pm}$are not identically zero. It suffices to multiply the solutions of the following root cases:

$$
\begin{aligned}
& \left.\begin{array}{l}
\chi^{+}(x)=1-\xi^{+} x \\
\chi^{-}(x)=1-\xi^{-} x
\end{array}\right\}, \quad \rho(x)=\frac{\left(\xi^{-} q x ; q\right)_{\infty}}{\left(\xi^{+} x ; q\right)_{\infty}} \\
& \left.\begin{array}{l}
\chi^{+}(x)=x^{m_{+}} \zeta^{+} \\
\chi^{-}(x)=x^{m_{-}} \zeta^{-}
\end{array}\right\}, \quad \rho(x)=\frac{g_{\gamma} h_{\gamma}}{g_{1} h_{1}}\left(g_{1} h_{1}\right)^{m}
\end{aligned}
$$

where $m=m_{-}-m_{+}$and $\gamma=(-1)^{m} \zeta^{-} q^{m_{-}} / \zeta^{+}$. Besides, Remark 4.4 provides infinitely many meromorphic solutions in $\mathbb{C} \backslash\{0\}$. For particular cases, choosing the simplest solution might not be completely trivial.

5. Taking $\chi^{ \pm}(x)=\left(1-\xi_{0}^{ \pm} x\right)\left(1-\xi_{1}^{ \pm} x\right)$ with $\xi_{0}^{ \pm} \neq 0$ and $\xi_{1}^{ \pm} \neq 0$, we obtain the $q$-Hahn polynomials

(a) $f_{k}(x)=f_{k}\left(1 / \xi_{0}^{-}\right){ }_{3} \phi_{2}\left(\begin{array}{c}q^{-k}, 1 / \xi_{0}^{-} x, q^{k-1} \xi_{0}^{+} \xi_{1}^{+} / \xi_{0}^{-} \xi_{1}^{-} \\ \xi_{0}^{+} / \xi_{0}^{-}, \xi_{1}^{+} / \xi_{0}^{-}\end{array} \mid q, q \xi_{1}^{-} x\right)$.

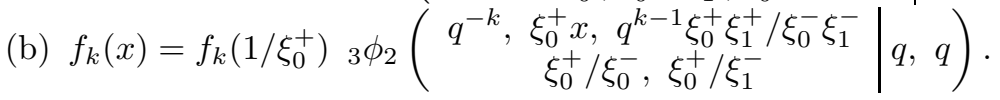


4.6. Trigonometric case. $\mathrm{P}(x, y)=x^{2}+y^{2}-2 a x y+a^{2}-1$ and $\theta_{t}=\frac{q^{t} u_{0}+q^{-t} u_{0}^{-1}}{2}$.

1. In this case, the Laurent polynomial $\chi(u)=\gamma_{-2} u^{-2}+\gamma_{-1} u^{-1}+\gamma_{0}+\gamma_{1} u+\gamma_{2} u^{2}$ provides the following parameterization:

$$
\begin{gathered}
\chi(u)=\sigma\left(\frac{u+u^{-1}}{2}\right)+\frac{\left(\lambda-\lambda^{-1}\right)\left(u-u^{-1}\right)}{4} \tau\left(\frac{u+u^{-1}}{2}\right), \\
\sigma\left(\frac{u+u^{-1}}{2}\right)=\frac{\chi(u)+\chi\left(u^{-1}\right)}{2} \text { and } \tau\left(\frac{u+u^{-1}}{2}\right)=2 \frac{\chi(u)-\chi\left(u^{-1}\right)}{\left(\lambda-\lambda^{-1}\right)\left(u-u^{-1}\right)} .
\end{gathered}
$$

2. We have $\alpha_{2}=2\left(\gamma_{2}+\gamma_{-2}\right), \beta_{1}=4\left(\gamma_{2}-\gamma_{-2}\right) /\left(\lambda-\lambda^{-1}\right)$ and

$$
\mu_{k}-\mu_{i}=4 \frac{\lambda^{i-k}-\lambda^{k-i}}{\left(\lambda-\lambda^{-1}\right)^{2}}\left(\gamma_{2} \lambda^{i+k-1}-\gamma_{-2} \lambda^{1-i-k}\right) .
$$

Taking $\theta_{t}=\left(q^{t}+q^{-t}\right) / 2$, we obtain $\widetilde{\sigma}^{ \pm}(t)=\chi\left(q^{ \pm t}\right)$ and $\widetilde{\sigma}_{j}^{ \pm}(t)=\chi\left(\lambda^{j} q^{ \pm t}\right)$. That is, the polynomials $\sigma_{j}$ and $\tau_{j}$ are parameterized by

$$
\chi_{j}(u)=\chi\left(\lambda^{j} u\right) .
$$

In particular, we have

$$
\tau_{j}\left(\frac{u+u^{-1}}{2}\right)=2 \frac{\chi\left(\lambda^{j} u\right)-\chi\left(\lambda^{j} u^{-1}\right)}{\left(\lambda-\lambda^{-1}\right)\left(u-u^{-1}\right)} .
$$

3. It suffices to consider the P-functions $\theta_{t}=\left(q^{t} u_{0}+q^{-t} u_{0}^{-1}\right) / 2$. In that case, we have $\widetilde{\sigma}^{-}(t)=\chi\left(q^{-t} u_{0}^{-1}\right)$. Thus, we take $u_{0}$ so that $\chi\left(u_{0}^{-1}\right)=0$. This condition provides the factorization $\chi(u)=\left(u-u_{0}^{-1}\right) u^{-2} \chi_{0}(u)$ for some $\chi_{0} \in \mathbb{P}_{3}[x]$. Therefore, it turns out that

Theorem 3.4 gives

$$
\tau_{i}\left(\theta_{i / 2}\right)=\frac{2 \chi_{0}\left(q^{i} u_{0}\right)}{\lambda^{3 i} u_{0}^{2}\left(\lambda-\lambda^{-1}\right)}
$$

$$
\partial_{j} f_{k}\left(\theta_{j / 2}\right)=\partial_{k} f_{k}\left[\begin{array}{c}
k \\
j
\end{array}\right]_{q} q^{-3\left[\left(\begin{array}{c}
k \\
2
\end{array}\right)-\left(\begin{array}{c}
j \\
2
\end{array}\right)\right]} \prod_{i=j}^{k-1} \frac{\chi_{0}\left(q^{i} u_{0}\right) / u_{0}^{2}}{2\left(\gamma_{2}-\gamma_{-2} q^{1-i-k}\right)} .
$$

Let us notice that, for $\chi(u)=\gamma_{ \pm 2} u^{ \pm 2}$, our methods provide a Rodrigues formula. However there is not any $u_{0}$ such that $\chi\left(u_{0}^{-1}\right)=0$. Hence we cannot apply Theorem 3.4. These cases correspond to the $q$-Hermite polynomials.

4. Now we apply the ideas of Section 4.4 to discuss some Rodrigues formulas for the trigonometric case when $|q|<1$. We begin by recalling that the relations

$$
\Omega=\left\{\frac{u+u^{-1}}{2}: u \in \Omega^{\prime}\right\} \quad \text { and } \quad \Omega^{\prime}=\left\{u: \frac{u+u^{-1}}{2} \in \Omega\right\}
$$

provide a bijective correspondence between the class of P-invariant open sets $\Omega$ of the complex plane and the class of $\lambda$-invariant open subsets $\Omega^{\prime}$ of $\mathbb{C} \backslash\{0\}$ satisfying that $u \in \Omega^{\prime}$ if and only if $u^{-1} \in \Omega^{\prime}$. Moreover, given $\Omega$ and $\Omega^{\prime}$ as above, the relation

$$
\mathrm{F}(u)=\mathrm{G}\left(\frac{u+u^{-1}}{2}\right)
$$

establishes a bijection between meromorphic functions $\mathrm{G}$ in $\Omega$ and meromorphic functions $\mathrm{F}$ in $\Omega^{\prime}$ satisfying $\mathrm{F}(u)=\mathrm{F}\left(u^{-1}\right)$. Consequently, we shall work with functions $\rho_{j}$ of the form

$$
\rho_{j}\left(\frac{u+u^{-1}}{2}\right)=h_{j}(u)
$$


where $h_{j}$ is meromorphic in $\Omega^{\prime}$ and $h_{j}(u)=h_{j}\left(u^{-1}\right)$ for all $u \in \Omega^{\prime}$. Taking the P-function $\theta_{t}=\left(q^{t}+q^{-t}\right) / 2$ with $u=q^{t}$, we have to solve

$$
\begin{aligned}
h(u) \chi(u) & =h(q u) \chi\left(q^{-1} u^{-1}\right), \\
h_{j+1}(u) & =h_{j}\left(\lambda^{\mp 1} u\right) \chi\left(\lambda^{j-1} u^{ \pm 1}\right) .
\end{aligned}
$$

We can always write the Laurent polynomial $\chi$ in the form

$$
\chi(u)=\zeta u^{-s} \prod_{\nu=0}^{r-1}\left(1-\xi_{\nu} u\right)
$$

where $r-s=\max \left\{k: \gamma_{k} \neq 0\right\}$ and $\zeta=\gamma_{k_{0}}$ such that $k_{0}=\min \left\{k: \gamma_{k} \neq 0\right\}$. Using the arguments of Section 4.4 and assuming $|q|<1$, we observe that the functions

$$
h_{j}^{\xi}(u)=\frac{1}{\left(\xi \lambda^{j} u ; q\right)_{\infty}\left(\xi \lambda^{j} / u ; q\right)_{\infty}}
$$

are meromorphic solutions in $\mathbb{C} \backslash\{0\}$ for $\chi(u)=1-\xi u$ and invariant under the change $u \mapsto u^{-1}$. On the other hand, when $\chi(u)=u^{-1}$ the system above reduces to

$$
\begin{aligned}
h(u) & =q u^{2} h(q u), \\
h_{j+1}(u) & =\lambda^{1-j} u^{ \pm 1} h_{j}\left(\lambda^{ \pm 1} u\right) .
\end{aligned}
$$

Let $h^{ \pm}(u)=\left(\mp \lambda^{1 / 2} u ; \lambda\right)_{\infty}\left(\mp \lambda^{1 / 2} / u ; \lambda\right)_{\infty}$. We have $h^{ \pm}(u)= \pm \lambda^{1 / 2} u h^{ \pm}(\lambda u)$. In particular, the functions $h^{+}$and $h^{-}$solve the functional equation $h(u)=q u^{2} h(q u)$. Moreover, if we define recursively the constants $k_{j}^{ \pm}$by

$$
k_{j+1}^{ \pm}= \pm \lambda^{\frac{1}{2}-j} k_{j}^{ \pm} \quad \text { with } \quad k_{0}^{ \pm}=1,
$$

it can be easily checked that the functions $h_{j}^{ \pm}=k_{j}^{ \pm} h^{ \pm}$are meromorphic solutions in $\mathbb{C} \backslash\{0\}$ for $\chi(u)=u^{-1}$ and invariant under the change $u \mapsto u^{-1}$. In summary, by Lemma 4.1 we have meromorphic solutions in $\mathbb{C} \backslash\{0\}$ of the system above for any Laurent polynomial $\chi$

$$
h_{j}(u)=\left(k_{j}^{ \pm} h^{ \pm}\right)^{s}(u) \prod_{\nu=0}^{r-1} h_{j}^{\xi_{\nu}}(u)=( \pm 1)^{j s} \lambda^{s\left[\frac{j}{2}-\left(\begin{array}{c}
j \\
2
\end{array}\right)\right]}\left(h^{ \pm}\right)^{s}(u) \prod_{\nu=0}^{r-1} h_{j}^{\xi_{\nu}}(u) .
$$

Therefore, the functions $\rho_{j}$ obtained by this process are meromorphic in the complex plane. For instance, if we have $s=2$ and $r=4$, we can choose the solution $h_{j}^{+} h_{j}^{-}$ for $\chi(u)=u^{-2}$ and so we get

$$
\rho_{j}\left(\frac{u+u^{-1}}{2}\right)=(-\lambda)^{j} q^{-\left(\begin{array}{c}
j \\
2
\end{array}\right)} \frac{\left(\lambda u^{2} ; q\right)_{\infty}\left(\lambda / u^{2} ; q\right)_{\infty}}{\prod_{\nu=0}^{3}\left(\xi_{\nu} \lambda^{j} u ; q\right)_{\infty}\left(\xi_{\nu} \lambda^{j} / u ; q\right)_{\infty}} .
$$

As in Remark 4.4 we can find other meromorphic solutions in $\mathbb{C} \backslash\{0\}$ of the given functional equation. Namely, it suffices to multiply $h$ by a function $f$ satisfying $f(\exp x)=g(x)$ with $g$ even, $2 \pi i$-periodic, $\log q$-periodic and meromorphic in $\mathbb{C}$.

Remark 4.8. The solutions we use for the functional equation $h(u)=q u^{2} h(q u)$ do not follow the general solution provided in Section 4.4. The main motivation for our election has been the simplicity of the resulting functions $\rho_{k}$. 
5. If $\gamma_{-2}$ and $\gamma_{2}$ are non-zero, we obtain the Askey-Wilson polynomials. Namely, normalizing if necessary, we can write

$$
\chi(u)=u^{-2} \prod_{\nu=0}^{3}\left(1-\xi_{\nu} u\right)
$$

in such a way that $\gamma_{-2}=1$ and $\gamma_{2}=\xi_{0} \xi_{1} \xi_{2} \xi_{3}$. Therefore we get

$$
f_{k}\left(\frac{u+u^{-1}}{2}\right)=f_{k}\left(\frac{\xi_{0}+\xi_{0}^{-1}}{2}\right){ }_{4} \phi_{3}\left(\begin{array}{c}
q^{-k}, \xi_{0} u, \xi_{0} / u, \xi_{0} \xi_{1} \xi_{2} \xi_{3} q^{k-1} \\
\xi_{0} \xi_{1}, \xi_{0} \xi_{2}, \xi_{0} \xi_{3}
\end{array} \mid q, q\right) .
$$

\section{Concluding Remarks}

1. In the canonical forms analyzed in Section 4 , the eigenfunctions are families of polynomials depending of five parameters which correspond to the coefficients of $\sigma$ and $\tau$. The number of free parameters can be reduced by considering two proportional hypergeometric operators to be equivalent. Moreover, recalling how $\mathrm{L}$ is transformed under the action of $\operatorname{Aff}(\mathbb{C})$

$$
g \mathrm{~L}^{\mathrm{P}} g^{-1}=\zeta^{2} \sigma^{g}\left(\mathrm{D}^{g \cdot \mathrm{P}}\right)^{2}+\zeta \tau^{g} \mathrm{~S}^{g \cdot \mathrm{P}} \mathrm{D}^{g \cdot \mathrm{P}},
$$

we can use the isotropy subgroups of the $\mathcal{A}_{1}$-orbits provided in Table I to identify different hypergeometric operators by suitable affine transformations. In particular, we can reduce the number of essential parameters in each canonical form. Namely, we have two free parameters in the continuous canonical form, three parameters in the arithmetic and geometric cases and four parameters in the quadratic and trigonometric canonical forms.

2. The Rodrigues formula given in 2] for the Askey-Wilson polynomials arise from (10) by taking

$$
\rho_{j}^{\mathrm{AW}}(u)=k(u) \rho_{j}\left(\frac{u+u^{-1}}{2}\right) .
$$

Here, the function $k$ is defined as follows:

$$
k(u)=\frac{\left(q u^{2} ; q\right)_{\infty}\left(q / u^{2} ; q\right)_{\infty}\left(u-u^{-1}\right)}{\left(\lambda u^{2} ; q\right)_{\infty}\left(\lambda / u^{2} ; q\right)_{\infty}} .
$$

The function $k$ is meromorphic in $\mathbb{C} \backslash\{0\}$ and satisfies the functional equation $k(q u)=k(u)$. However, we have $k(u)=-k\left(u^{-1}\right)$. Therefore, the functions used in [2] are ramified in the variable

$$
x=\frac{1}{2}\left(u+u^{-1}\right) .
$$

The function $k$ can be easily regarded in terms of Jacobi's $s n$ elliptic function.

3. Let us consider a function $\varepsilon$ meromorphic in $\mathbb{C} \backslash\{0\}$ and such that

○ The function $\varepsilon$ satisfies $\varepsilon(u)=\varepsilon(q u)=-\varepsilon(1 / u)$.

○ The function $\varepsilon(\lambda u) \rho_{1}\left(\frac{1}{2}\left(u+u^{-1}\right)\right)$ is regular for $\lambda \leq|u| \leq \lambda^{-1}$.

Then it can be proved that, assuming that $q, \xi_{\nu} \in(0,1)$ and adapting the arguments of [14, 3.10.5.2], the hypergeometric operator $\mathrm{L}$ is symmetric with respect to the product

$$
\langle f, g\rangle=\int_{-1}^{1} f(x) g(x) \rho(x) \widetilde{\varepsilon}(x) d x,
$$

where $\widetilde{\varepsilon}(\cos (s))=\varepsilon\left(e^{i s}\right)$ for $0 \leq s \leq \pi$. This gives the orthogonality relations for the Askey-Wilson polynomials $f_{k}$. The function $k$ defined on the previous point 
satisfies the required conditions on $\varepsilon$. In fact, by the Riemann-Roch theorem, it can be checked the it is unique up to a constant factor. Hence, this allows us to recover the well-known orthogonality relations given in 2 .

\section{ACKNOWLEDGMENTS}

The authors thank Gabino González for a useful conversation on the RiemannRoch theorem. We also thank the referee for some interesting comments and for having brought some references to our attention. After this paper was accepted for publication, Paul Terwilliger communicated to us the close relation existing between this paper and the notion of a Leonard pair. We refer the reader to Terwilliger's paper [16] for more on this topic. This research was supported in part by MCYT Spain via the Project BFM 2001/0189.

\section{REFERENCES}

1. G.E. Andrews, R. Askey and R. Roy, Special Functions, Encyclopedia of Mathematics and its Applications 71, Cambridge Univ. Press, 1999. MR2000g:33001

2. R. Askey and J.A. Wilson, Some basic hypergeometric orthogonal polynomials that generalize Jacobi polynomials, Mem. Amer. Math. Soc. 54 (1985), no. 319. MR.87a:05023

3. G. Bangerezako, The factorization method for the Askey-Wilson polynomials, J. Computational and Applied Math. 107 (1999), 219-232. MR2000h:33015

4. E. Bannai and T. Ito, Algebraic Combinatorics I: Association Schemes, Benjamin/Cummings Publishing Co., Inc., Menlo Park, 1984. MR87m:05001

5. A.E. Brouwer, A.M. Cohen and A. Neumaier, Distance regular graphs, Springer-Verlag, 1989. MR.90e:05001

6. B.M. Brown and M.E.H. Ismail, A right inverse for the Askey-Wilson operator, Proc. Amer. Math. Soc. 123 (1995), 2071-2079. MR.95i:33019

7. I. Fischer, A Rodrigues-Type formula for the $q$-Racah polynomials and some related results, Contemporary Mathematics 169 (1994), 253-259. MR95j:33049

8. G. Gasper and M. Rahman, Basic hypergeometric series. With a foreword by Richard Askey, Encyclopedia of Mathematics and its Applications 35, Cambridge Univ. Press, 1990. MR91d:33034

9. D.A. Leonard, Orthogonal polynomials, duality and association schemes, SIAM J. Math. Anal. 13 (1982), 656-663. MR83m:42014

10. J.H. van Lint and R.M. Wilson, A course in combinatorics, Cambridge Univ. Press, 1992. MR.94g:05003

11. A.P. Magnus, Associated Askey-Wilson polynomials as Laguerre-Hahn orthogonal polynomials, Lecture Notes in Math., vol. 1329, Springer, Berlin, 1986, pp. 261-278. MR90d:33008

12. J.M. Marco and J. Parcet, On the natural representation of $S(\Omega)$ into $L^{2}(\mathcal{P}(\Omega))$ : discrete harmonics and Fourier transform, J. Combin. Theory Ser. A 100 (2002), 153-175. MR.2003m:05209

13. J.M. Marco and J. Parcet, Taylor series for the Askey-Wilson operator and classical summation formulas. Submitted for publication.

14. A.F. Nikiforov, S.K. Suslov and V.B. Uvarov, Classical orthogonal polynomials of a discrete variable, Springer Series in Computational Physics, Springer-Verlag, Berlin, 1991. MR 92m:33019

15. M. Rahman and S.K. Suslov, The Pearson equation and the beta integrals, SIAM J. Math. Anal. 25 (1994), 646-693. MR95f:33001

16. P. Terwilliger, Two linear transformations each tridiagonal with respect to an eigenbasis for the other, Linear Alg. Appl. 330 (2001), 149-203. MR2002h:15021

Department of Mathematics, Universidad Autónoma de Madrid, Madrid 28049, Spain

Department of Mathematics, Universidad Autónoma de Madrid, Madrid 28049, Spain E-mail address: javier.parcet@uam.es 